San Jose State University

SJSU ScholarWorks

Master's Theses

Master's Theses and Graduate Research

2003

\title{
Surface Enhanced Raman Scattering of 4-Mercaptobenzoic [sic] acid in silver colloid
}

Leonard Edward Jusinski

San Jose State University

Follow this and additional works at: https://scholarworks.sjsu.edu/etd_theses

\section{Recommended Citation}

Jusinski, Leonard Edward, "Surface Enhanced Raman Scattering of 4-Mercaptobenzoic [sic] acid in silver colloid" (2003). Master's Theses. 2527.

DOI: https://doi.org/10.31979/etd.karr-zry7

https://scholarworks.sjsu.edu/etd_theses/2527

This Thesis is brought to you for free and open access by the Master's Theses and Graduate Research at SJSU ScholarWorks. It has been accepted for inclusion in Master's Theses by an authorized administrator of SJSU ScholarWorks. For more information, please contact scholarworks@sjsu.edu. 
SURFACE ENHANCED RAMAN SCATTERING OF 4-MERCAPTOBENZOIC ACID IN SILVER COLLOID

\author{
A Thesis \\ Presented to \\ The Faculty of the Department of Physics \\ San Jose State University
}

\begin{abstract}
In Partial Fulfillment
of the Requirements for the Degree

Master of Physics
\end{abstract}

by

Leonard Edward Jusinski

December 2003 
UMI Number: 1419669

Copyright 2003 by

Jusinski, Leonard Edward

All rights reserved.

\title{
INFORMATION TO USERS
}

The quality of this reproduction is dependent upon the quality of the copy submitted. Broken or indistinct print, colored or poor quality illustrations and photographs, print bleed-through, substandard margins, and improper alignment can adversely affect reproduction.

In the unlikely event that the author did not send a complete manuscript and there are missing pages, these will be noted. Also, if unauthorized copyright material had to be removed, a note will indicate the deletion.

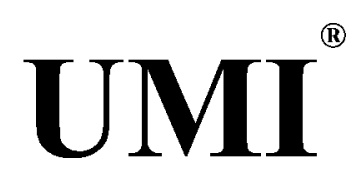

UMI Microform 1419669

Copyright 2004 by ProQuest Information and Learning Company. All rights reserved. This microform edition is protected against unauthorized copying under Title 17, United States Code.

\author{
ProQuest Information and Learning Company \\ 300 North Zeeb Road \\ P.O. Box 1346 \\ Ann Arbor, MI 48106-1346
}


$\odot 2003$

Leonard Edward Jusinski ALL RIGHTS RESERVED 
APPROVED FOR THE DEPARTMENT OF PHYSICS
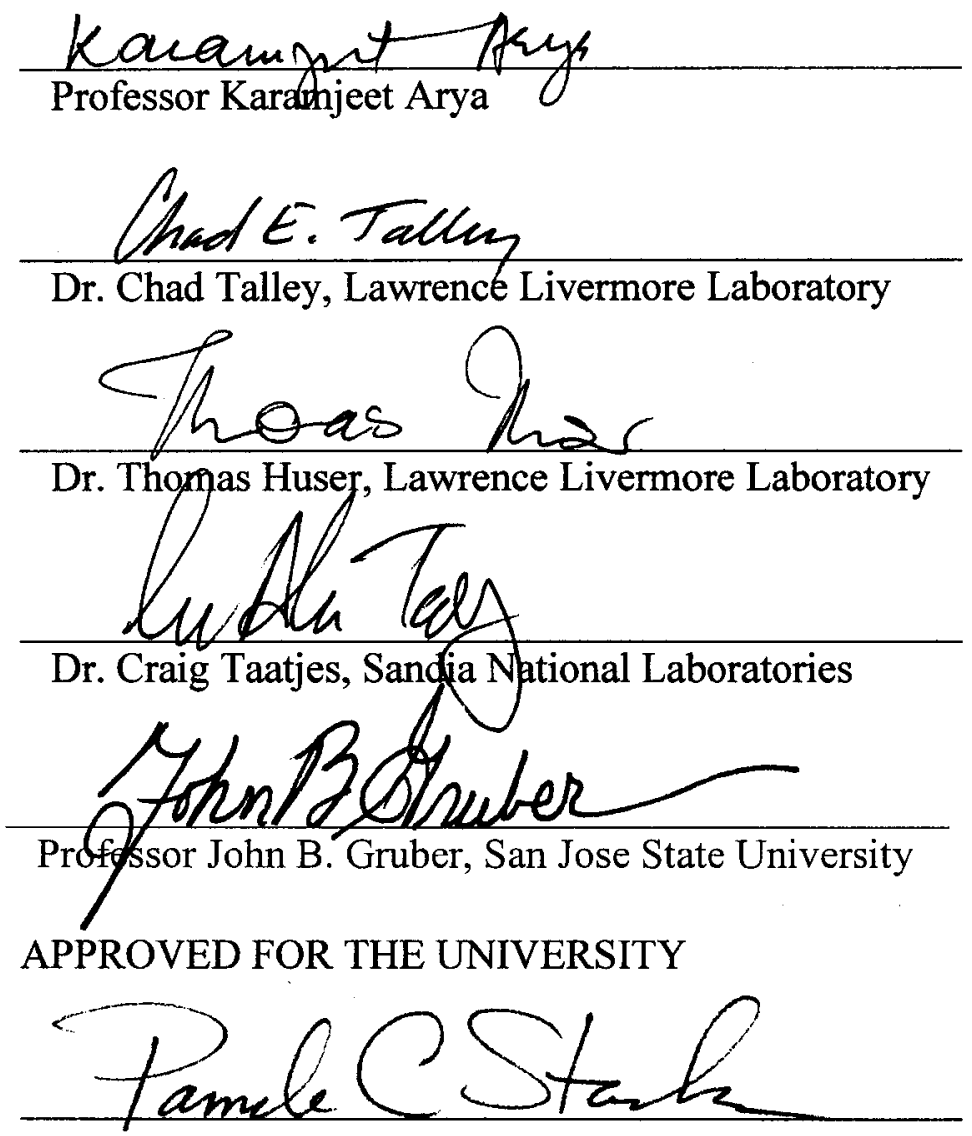


\begin{abstract}
The ionic strength and $\mathrm{pH}$ dependence of Surface Enhanced Raman Scattering (SERS) of p-Mercaptobenzoic acid (pMBA) in silver colloid has been investigated. Disassociative adsorption of pMBA at surfaces of the silver colloid was found to occur via both its sulfur and carboxylate groups. Titration curves at low (few $\mathrm{mM})$ and high (100mM) ionic strengths indicated significantly different ionization coefficients of the carboxylate ion on colloidal silver particles. In low ionic strength solution, pMBA existed as p-sulphidobenzoate in basic solution at pH 8 and above. At pH 4, complete protonation of the carboxylate group had occurred and pMBA existed as p-sulphidobenzoic acid. In higher ionic strength $(100 \mathrm{mM})$, complete protonation of the carboxylate group occurred at pH 11 and the solution existed as p-sulphidobenzoic acid during further acidification down to $\mathrm{pH} 2$. Aggregation effects of colloidal silver solutions could possibly explain this anomalous behavior.
\end{abstract}


For my Parents and Family 


\section{Acknowledgements}

This work was supported by...

\section{San Jose State University}

Department of Physics

\section{Lawrence Livermore National Laboratory \\ Medical Technology Proram}

\section{Sandia National Laboratories}

Graduate Degree Program 


\section{Table of Contents}

I. Introduction ............................................. 1

II. Theory ...................................................... 12

III. Experimental Apparatus ................................... 29

IV. Results and Discussion ................................... 36

V. Conclusions ............................................... 42

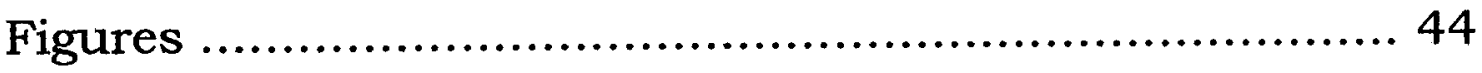

References ............................................................. 59 


\section{Introduction}

Raman spectroscopy is an analytical optical technique used to study the vibrational spectrum of molecules in liquid or gas phase. Raman scattering is an inelastic process, where an incident photon, usually in the visible wavelength region, excites a molecule to a higher vibrational state. The photon is then scattered with a lower energy equal to the energy of the excited vibration. This process is called Stokes Raman scattering [1, 2]. A graphical representation of this process is shown in Figure 1.

At finite temperatures, there is a finite probability that a molecule may be in an excited vibrational state. According to Boltzmann statistics, this probability is proportional to the absolute temperature and the energy of the vibrational transition. An incident photon can then interact with the molecule leaving it in a lower vibrational state, absorbing the vibrational energy in the process. The incident photon now has a higher energy than when it arrived. This process is known as anti-Stokes Raman scattering.

Raman scattering is a weak process. Typical cross sections are on the order of $10^{-30} \mathrm{~cm}^{2}$ per molecule. In liquids, even though there are approximately $10^{24}$ molecules per $\mathrm{cm}^{3}$, the Raman signal is still very weak. It is surprising that in his first experiment, using only a mercury lamp, a flask of benzene, and a direct vision pocket spectroscope, C.V. 
Raman could observe a vibrationally shifted spectrum.[3] This landmark experimental measurement confirmed earlier predictions of quantum mechanics. For his invention, Professor Raman was awarded the Nobel Prize in Physics in 1930, which is now known as the Raman effect. Historically, Raman scattering was mainly used for the study of molecules in liquid or gas phase due to the inefficiency of the process. With the advent of the laser and the ability to deliver a high spectral density of photons, Raman scattering is now used to study excitations in solids, such as phonons, magnons, and excitons. [4]

For a molecule with $\mathrm{N}$ atoms, there are $3 \mathrm{~N}-5$ normal modes for a linear geometry and 3N-6 otherwise.[1] For example, water, H2O, having three atoms, has 3 normal mode vibrations. As the number of atoms in a molecule increases, the number of vibrational modes also increases. The vibrational spectrum becomes increasingly complicated. This complication does not become unmanageable since molecules form in similar structures, for example, aromatic ring structures, functional groups, and so on. These modalities provide similar vibrational spectra. Therefore, the interpretation of spectra containing different functional groups becomes a matter of identifying familiar segments of the entire spectral fingerprint of the molecule.

In Raman scattering, a molecule becomes polarized due to the electric field of the incident photon. [2] Photons are scattered by 
molecules when the photon induces a change in the molecular polarization. The response of the molecular polarization to the electric field of the photon is known as the susceptibility $X$. When a vibrational mode results in a change in polarizability, this mode is referred to as "Raman active".

Not all vibrations are Raman active. Complimentary to the Raman activity is infrared activity (IR). IR activity is a result of a photon inducing a change in dipole moment in the molecule. When a change in dipole moment occurs, a photon can be absorbed, resulting in IR absorption. The energies involved in these transitions are on the order of hundreds of wavenumbers, and thus are in the IR electromagnetic spectrum. Depending on the particular vibrational mode, a vibration can be both infrared and Raman active.

Vibrational spectroscopy is a combination of both Raman and IR activity. Their complementary nature yield a wealth of information about the structure of a molecule. There are many variations on how one can excite a vibration, and also how one can observe the resulting scattering process.

Raman spectroscopy is therefore a very powerful analytical technique. However, because of its inherently low scattering efficiency, only with the advent of the laser did the technique become a very popular and widely used analytical instrument. In a highly coherent source such 
as the laser, the density of modes, or the number of photons in a given frequency range, is extremely high.[5] Therefore, even though the chance of an incident photon being inelastically scattered is very low, the large number of incident photons provide an adequate number of scattered photons capable of detection. Since the Raman scattered intensity is a linear function of the incident intensity, the more power the laser has, the better. Conventional lasers can provide this needed intensity.

In contrast to absorption, the Raman process can occur with any wavelength source. Absorption requires an energy match between the exciting source and the vibrational transition. In order to observe infrared absorption, infrared sources are required. Raman scattering does not have this restriction. The scattering source can be virtually any wavelength, with the scattered photon either loosing or gaining one or more vibrational quanta.

With this new instrument in the experimentalists' toolbox, more detailed studies could now be performed using vibrational spectroscopy.[6] The laser has two important properties that could now be exploited. First, the frequency bandwidth of the light is very narrow, allowing much higher resolution studies to be performed. Second, the energy fluence in this narrow bandwidth, the number of photons traveling through a given area in a given time, is very high. Even with 
these advantages, a large number of molecules are needed in order to measure normal Raman scattered photons.

In addition to the availability of high power lasers with frequencies extending continually from the ultraviolet to the infrared, the development of low noise, high sensitivity detectors with high quantum efficiency have become available. $[2,5]$ These include intensified charge coupled arrays (ICCD) having high spatial resolution for imaging applications, and avalanche photo diodes (APD) for single frequency detection. Increased sensitivity has also been realized by the development of high throughput holographic transmission and rejection filters.

Another advantage of the Raman process is its ability to provide noninvasive molecular information without sample preparation. Light can be delivered and collected remotely allowing analysis of regions inaccessible with normal materials. Applications include the analysis of flames, the remote detection of pollutants, or the detection of biologically relevant molecules, in particular, glucose.[2, 6-18]

Nearly thirty years ago it was observed that when a roughened metal surface is used as a substrate for Raman scattering, a large enhancement in the scattering efficiency is observed. Enhancements in scattering efficiency on the order of six to eight orders of magnitude were easily obtained, and readily duplicated in various laboratories. [19] This 
finding resulted in a new excitement concerning the analytical capabilities of the Raman process.

In 1974, Fleischmann observed intense Raman scattering from pyridine adsorbed on a roughened silver electrode in aqueous solutions. [20]. This enhancement was initially thought to be due to an increase of adsorbed molecules on the roughened metal surface, since the rough surface now provided an increase in surface area. Several other laboratories could duplicate these results, but disagreed with the conclusion the enhancement was simply due to an increase of adsorbed molecules. $[13,19,21-27]$ It was proposed the enhancement was due to the excitation of surface plasmons on the metal surface and to a much smaller extent, to the adsorbed state of the molecule. [28-30] The debate over the SERS mechanism continues today.

Although controversy still exists on the exact nature of the enhancement, it is generally agreed the SERS effect is dominated by two processes, an electromagnetic enhancement and a chemical enhancement.

The electromagnetic enhancement theory has been treated using particles of various sizes and shapes. $[7,21,28,29,31-39]$ Spheres and ellipsoids, whether they be isolated or interacting, have been theoretically developed. The simplest treatments use an electrostatic approximation 
with sharp boundaries whose solutions are calculated numerically. Further developments include the effects of dielectric overlayers. [32]

The theory of chemical enhancement, first introduced by Otto, involves a charge transfer mechanism where new electronic states are produced by chemisorptions to the metal surface. $[7,40]$ These new electronic states can be shifted and broadened by the adsorbate providing an avenue for resonant enhancement.

Laboratories have reported single molecule detection using SERS. $[9,12,13,41,42]$ The rich information provided by Raman spectroscopy combined with the single molecule detection sensitivity has yielded the capability of observing single events without the effects of ensemble averaging, thus providing basic scientific information into the nature of the physical event.

Even though the strong SERS effect is of electromagnetic origin, the enhancement is not observed for all molecules. There continues to exist a molecular sensitivity which has yet to be explained. Empirical experimental measurements have shown large SERS enhancements for molecules containing delocalized pi electronic systems[14]. This finding provides hope for SERS application for the detection of biological molecules since many molecules of biomedical and pharmaceutical interest contain unsaturated cyclic electron systems. 
Conventional single molecule techniques are mainly based on laser induced fluorescence using optical tags. Fluorescence Resonant Energy Transfer (FRET) is one such technique.[43, 44] Although these fluorescent techniques provide high sensitivity, the information provided, especially at room temperature, is limited. The detection of a single molecule with its unique molecular structure and the capability to observe its dynamic behavior in a variety of environments is now possible.

Many fields can now benefit from this capability. The study of biological molecules such as DNA can now be performed on single base pair, furthering the progress in human DNA sequencing. $[12,18]$ Pharmacological development can also benefit from the observation of single cell interactions with biological membranes. Single DNA fragments can now be identified and be of great interest in the field of medicine. $[12,14]$

One problem that has slowed the development of SERS has been the reproducibility of the enhanced Raman scattering. Although the chemical enhancement does play a minor role, the dominant issue to se solved is the reproducibility of the geometry of the SERS substrate. With the advent of nanotechnology and the ability to produce geometrically similar substrates at the nanoscale, reproducible substrates are now being produced. [45] In addition to reproducible substrates of the 
spherical geometry, substrates in the shape of nanoprisms have been fabricated with the possibility of increasing even further the local electric field. [46] Colloids have also been prepared which possess a low degree of turbidity to allow use in optically challenged applications. [30, 47]

Another application of SERS is the use of silver or gold spheres functionalized with environment sensing molecules. [15, 48, 49] PMBA is one such molecule. Since the binding of pMBA to gold and silver surfaces has been shown to occur primarily via the sulphur group at both low and high $\mathrm{pH}$, the ionization of the carboxylate group could be an indicator of the surrounding $\mathrm{pH}$. The addition of pMBA functionalized metal particles into a solution or even single cell could provide information on the local environment. Kneipp et al has injected unfunctionalized gold nanoparticles into single cells and has obtained SERS spectra of DNA base pairs.[50] The use of functionalized nanoparticles could be prepared to provide specific binding to the molecule of interest eliminating the spectra from unwanted adsorbed species.

In addition to the use of a functionalized colloid, self-assembled monolayers (SAM) could be prepared on a larger metal surface, which would also provide molecular specificity. $[13,15,16]$ Molecules sensitive to the carboxylate functional group could be detected using a SAM prepared with pMBA. [48] 
PMBA could also provide the linking properties necessary to obtain SERS spectra of molecules without the ability to adsorb directly to metals, such as glucose. [16] Linker molecules have one functional group, such as a thiol, to provide adsorption to a metal surface, with another functional group capable of forming a complex with the molecule of interest. In this way, SERS spectra can be obtained of the desired analyte via the linker molecule. Little has to be mentioned about the importance and utility of non-invasive glucose monitoring. Patient conformation to diabetic therapy, which presently uses methods that are invasive and painful, would benefit from a method that is simple and painless.

This thesis will investigate the ionic strength and $\mathrm{pH}$ dependent spectra of p-Mercaptobenzoic acid in silver colloid. Section 2 will discuss the theory of both normal and surface-enhanced Raman scattering. The theory of Mie will be applied to scattering of colloidal silver particles. The double layer potential on surfaces in ionic solutions will be presented using the Guoy-Chapmann model. Section 3 will discuss the experimental apparatus and methods of sample preparation. Section 4 will present the experimental results and discussion of the findings. Finally, Section 5 will present the conclusions and discuss the future directions of this study. The potential of using this technique for the real 
time observation of the formation of Self Assembled Monolayers (SAMS) will be proposed. 


\section{Theory}

Raman scattering is an inelastic collision of an incident photon $\hbar \omega_{i}$ with a molecule in an initial energy level $\mathrm{E}_{\mathrm{i}}$. Following the collision, photon $\hbar \omega_{s}$ is emitted and the molecule is found in a higher energy level $E_{\text {f. }}$

The development of the absorption, emission, and scattering of light starts with the assumption of the behavior of an oscillating dipole. When light is incident on a molecule, one of the possible interactions is an inelastic scattering known as the Raman effect. The oscillating electric field of the incoming light induces a polarization of the electrons in the molecule and produces an oscillating dipole. This induced dipole then radiates scattered light. If there is no change in energy between the incident and scattered photon, this effect is known as Rayleigh scattering.

There is a small probability the incident photon can exchange energy with molecule. When this energy is quantized in units of vibrational quanta, the Raman effect is observed.

The induced dipole moment of the molecule can radiate energy with or without the exchange of energy. The strength of the induced polarization is given by

$$
\mathbf{P}=\tilde{\alpha} \mathbf{E}
$$


where $\mathrm{P}$ is the induced polarization, $\mathrm{a}$ is the polarizability, and $\mathrm{E}$ the incident electric field of the photon.

This fundamental relationship applies to both classical and quantum mechanical treatment of induced polarization and Raman scattering. This relationship is the basis for the development of the Raman cross section, which is the relavent analytical parameter most commonly referred to in experimental measurements.

Classically, the effects of the molecular vibrations on the polarizability can describe the Raman scattering. Assume the incident photon electric field is described by the relationship

$$
\mathbf{E}=\mathbf{E}_{o} \cos 2 \pi v_{o} t
$$

where $E_{o}$ is the amplitude of the electric field and $v_{o}$ the frequency of the incident photon.

The classical treatment of the vibrational Raman effect assumes the form of the complete dipole moment. Assume the complete dipole moment can be written in the form

$$
\mathbf{p}=\boldsymbol{\mu}_{0}+\tilde{\alpha} \mathbf{E}
$$


where $\mathbf{p}$ is the total dipole moment, $\boldsymbol{\mu}_{0}$ is the possible permanent moment of the molecule, $\alpha$ is the polarizability tensor of rank two. The molecular vibrations of the system can be considered as consisting as a series of vibrational eigenmodes. For a linear molecule there are $3 N-5$ normal vibrational modes, where $N$ is the number of atoms. For molecules consisting of more than three atoms, and for nonlinear molecules, there are $3 \mathrm{~N}-6$ normal eigenmodes of vibration.

The molecule can then be considered as consisting as a series of $Q_{j}$ normal vibrational modes consisting of $\mathrm{N}$ atoms. Individually these vibrations can be represented as

$$
\mathbf{Q}_{j}=\mathbf{Q}_{0 j} \cos 2 \pi v_{j} t
$$

where $v_{j}$ is the characteristic harmonic frequency of the $j$ th normal mode and $\boldsymbol{Q} j$ is the $j$ th displacement coordinate.

The electric field of the incident photon will modulate the polarizability of the electrons in the molecule. The dipole moment and polarizability are functions of the coordinates of the electrons and nuclei.

Two assumptions must be made to continue this classical development. If the frequency of the incident photon is significantly far off resonance with the vibrational transition, the nuclear displacements 
induced by the polarization of the electron cloud should be small.

Second, we assume the electron cloud adjusts "instantaneously" and the much heavier nucleus does not have time to move and adjust to the charge redistribution. With these assumptions the dipole moment and polarizability can be expanded into a Taylor series in the normal coordinates $\mathrm{q}_{\mathrm{j}}$ of the nuclear displacements.

The molecular polarizability can then be expanded in the form

$$
\tilde{\boldsymbol{\alpha}}=\tilde{\mathbf{a}}_{0}+\left(\frac{\delta \tilde{\boldsymbol{\alpha}}}{\delta \mathbf{Q}_{j}}\right) \mathbf{Q}_{j}+\cdots
$$

It is useful at this point to make use of the trigonometric identity, which relates the product of two cosines to the cosine of the sum of the two variables. Noting that

$$
\cos a \cos b=\frac{\cos (a+b)+\cos (a-b)}{2}
$$

the complete dipole moment can now be written in an intuitive form. Keeping terms up to the second order, the polarization $\mathbf{P}$ can now be written as 


$$
\mathbf{P}=\alpha_{0} E_{0} \cos 2 \pi v_{0} t+E_{0} Q_{0_{j}}\left(\frac{\delta \alpha}{\delta Q_{j}}\right) \frac{\cos 2 \pi\left(v_{0}+v_{j}\right)+\cos 2 \pi\left(v_{0}-v_{j}\right)}{2}
$$

Inspection of this equation reveals the behavior of the oscillating induced polarization of the molecule. It is shown that the incident oscillating electric field of the photon will cause the polarized electrons of the molecule to radiate and scatter at three distinct frequencies.

The first term is referred to as Rayleigh scattering, where the frequency of the scattered radiation does not change from the incident photon frequency. In other words, the photon is scattered inelastically and there is no transfer of energy from the incident photon to the scattering molecule. The magnitude of the Rayleigh scattered frequencies are directly proportional to the inherent polarizability of molecule.

The second and third term show the production of scattered frequencies which differ from the incident photon. The magnitude of this difference is one vibrational quanta of the jth vibrational mode. In other words, there has been an exchange of energy between the incident photon and the molecule. This exchange of energy is referred to as an inelastic scattering process.

In terms of Raman theory, if the scattered photon gains a vibrational quanta, its frequency and energy increases, and is referred to 
as anti-Stokes radiation. If the scattered photon looses a vibrational quanta to the molecule, its frequency and energy decreases, and is referred to as Stokes radiation.

The selection rules for Raman and IR activity can be briefly summarized. [33] Qualitatively, a vibrational mode is IR active when there is a change in the molecular dipole moment during the vibration. A vibrational mode is Raman active when there is a change in the molecular polarizability during the vibration. Qualitatively, a vibrational mode, which is antisymmetric, or a molecular vibration resulting from polar groups tends to exhibit stronger IR absorption. Vibrational modes resulting from symmetric oscillations tend to exhibit stronger Raman scattering intensities. IR and Raman intensities are mutually exclusive for molecules possessing a center of symmetry.

A word about Raman nomenclature is worth noting here. When one speaks of "characteristic frequencies" of Raman bands, the reference is used to describe the Raman bands common for molecules containing the same functional groups. These characteristic frequencies are then further broken down into group frequencies as the vibrational band produced by a submolecular group of atoms in a characteristic spectral region. These broup frequencies fall into three basic types. These consist of those having a rather constant frequency regardless of the environment, those that move around according to their molecular 
environment in a somewhat predictable manner, and those that have unpredictable positions or intensities.

This classical treatment of the theory does provide important insights into the process, but it is incomplete. This development does predict the scattering frequencies of the Raman lines, but it does not predict the intensities. A quantum mechanical treatment is necessary to predict the intensities.

The classical treatment does provide several intuitive observations and useful insights about the nature of the Raman scattering process.

First of all, it is shown the polarization and scattering intensities of both the Rayleigh and Raman frequencies are linear with the laser intensity. Of course, non-linear effects can occur at extremely high electric fields, but are not an issue with the measurements either of this thesis or in analytical applications. Non linear effects using extremely high electric field are typically used to generate additional frequencies as probes for other physical processes, such as Laser Induced Fluorescense (LIF) or Multi Photon Ionization (MPI).

The basic selection rule for the production of Raman scattering is shown. In order to produce Raman frequencies, there must be a change in the polarizability of the molecule. The first derivative of the polarizability with respect to the normal coordinate, $\left(\frac{\tilde{\delta} \tilde{\boldsymbol{\alpha}}}{\delta \mathbf{Q}_{j}}\right)$, must not be 
equal to zero. This partial derivative is the fundamental variable, which determines the cross section of the relevant vibration. The development of the Raman cross section will be developed in more detail in the following paragraphs.

Raman shifts may be of either higher or lower frequency. The intensity of the shifts depends on the population of the vibrational levels. The temperature through the Boltzman distribution governs the vibrational level populations. Assuming nondegenerate vibrations, the Raman intensities are given by [2]

$$
\frac{I_{R}\left(v_{0}+v_{j}\right)}{I_{R}\left(v_{0}-v_{j}\right)}=\frac{\left(v_{0}+v_{j}\right)^{4}}{\left(v_{0}-v_{j}\right)^{4}} \exp \left(\frac{-h v_{j}}{k T}\right)
$$

Large variations in normal mode vibrational band intensity for a given molecule can vary significantly since the intensity of the Raman scattered frequencies are proportional to the spatial derivative of the polarizability $\left(\frac{\delta \tilde{\boldsymbol{\alpha}}}{\delta \mathbf{Q}_{j}}\right)$. These variations occur not only by spontaneous, or normal, Raman systems, but can be significantly effected by chemical or surface enhanced mechanisms. [33, 35]

Though not evident from the classical form of the complete mathematical form of the dipole moment, Raman scattering is much 
weaker than Rayleigh scattering, since $\left(\frac{\delta \tilde{\boldsymbol{\alpha}}}{\delta \mathbf{Q}_{j}}\right)$ is generally much smaller than ao.

The intensity of Raman scattering is proportional to the Raman cross-section, $\sigma_{\mathrm{j}}$, and has the units of square centimeters per molecule. Since the Raman scattering is a result of the oscillating molecular dipole of the $\mathrm{j}^{\text {th }}$ vibration, the cross section is proportional to the quantity $\left(\frac{\delta \tilde{\boldsymbol{\alpha}}}{\delta \mathbf{Q}_{j}}\right)$. The development of the cross section requires a rigorous theoretical treatment and excellent reviews are available for the interested reader. $[31,33,35]$ Summaries of the results are presented here.

A significant feature of the rigorous development is the relationship between the Raman intensity and its associated frequency. This relationship can be expressed as

$$
I_{R}=\mu\left(v_{0} \pm v_{j}\right)^{4} \alpha_{j}^{2} Q_{j}^{2}
$$

where $\mu$ is a constant.

Raman spectroscopy is a process where a shift in energy, or wavelength, occurs. Since this shift in energy is independent of the wavelength of the incident photon, it is more conventional to express 
Raman frequencies as shifts in units of reciprocal centimeters, or wavenumbers. This relationship is expressed as

$$
\bar{v}=\frac{v}{c}=\lambda^{-1}
$$

where $c$ is the speed of light and $\lambda$ is the wavelength.

A complete definition of the Raman cross section provides the rigorous treatment of the total scattered light scattered in the complete $4 \pi$ steradian solid angle. In practical applications, this is not a very useful quantity. Although one should pay attention to the effects of the incident photon polarizability, wavelength, and other relevant physical properties which determine the Raman intensity, it more useful in analytical applications to use an empirically determined cross section which depends upon the geometry of the apparatus. The use of a single cross section for a given laser wavelength and observation geometry is arguably a major simplification of reality, but this pragmatic approach is usually both adequate and desirable for the required analytical application.

The scattered Raman intensity expressed in watts (joules/sec) is related to the Raman cross-section and the incident photon intensity through the relationship 


$$
I_{R}=I_{0} \sigma_{j} N d z
$$

where $\mathrm{N}$ is the number of molecules per volume and $\mathrm{dz}$ is the interaction path length. In practice, the actual interaction length might be a difficult parameter to accurately determine. A reference measurement would be most useful since $\mathrm{dz}$ might be difficult to obtain for a given optical collection geometry.

The scattering cross section can be determined by using third order time dependent perturbation theory. The three orders correspond to two orders in electron-photon interaction, which represent the incident and scattered photon. One additional order represents the electron-vibration interaction. Briefly summarized here are the calculations for normal Raman scattering cross-sections followed by those representing surface-enhanced Raman scattering.

The development of third order perturbation theory starts with the total Hamiltonian for the system. The total Hamiltonian can be written as

$$
\mathbf{H}=\mathbf{H}_{0}+\mathbf{H}_{e-p_{i}}+\mathbf{H}_{e-v}+\mathbf{H}_{e-p_{s}}
$$


where $\mathrm{H}_{0}$ is the unperturbed Hamiltonian for the molecule, $\mathrm{H}_{\mathrm{e}-\mathrm{p}}$ is the perturbation for the incident and scattered photon, and $\mathrm{H}_{\mathrm{e}-\mathrm{v}}$ is the perturbation for the electron-vibration interaction.

The Hamiltonian for the dipole interaction can be written as

$$
\mathbf{H}_{e p}=-e \mathbf{r} \cdot \mathbf{E}\left(\omega_{i}\right) e^{-i \omega_{i} t}
$$

where $\mathrm{e}$ is the electron charge, $\mathbf{r}$ is the spatial coordinate, and $\mathbf{E}\left(\omega_{i}\right)$ is the electric field corresponding to the incident scattered photon.

In Raman scattering, the electron occupies the same initial and final electronic states, but differ only in the vibrational level. Using time dependent perturbation theory, one can write the transition rate for the molecule in the initial state $|i, 0\rangle$ to be excited to a final state $|i, 1\rangle$ as

$$
W \approx \frac{2 \pi}{\hbar}\left|\frac{\left\langle i, 1\left|H_{e p}\right| m, 1\right\rangle\left\langle m, 1\left|H_{e v}\right| m, 0\right\rangle\left\langle m, 0\left|H_{e p}\right| i, 0\right\rangle}{\left(E_{m}-E_{i}-\hbar \omega_{i}\right)\left(E_{m}-E_{i}-\hbar \omega_{s}\right) \delta\left(E_{i 1}-E_{i 0}-\hbar\left(\omega_{i}-\omega_{s}\right)\right)}\right|^{2}
$$

This equation corresponds to the dominant contribution in the Stokes Raman process. A complete calculation must include the phase space, or number density of modes in a unit volume. The phase space is given by 


$$
\left(\frac{V \omega_{s}^{2}}{8 \pi^{3} c^{3}}\right) d \omega_{s} d \Omega
$$

where $V$ is the volume, $\omega_{s}$ is the scattered stokes frequency, $c$ is the speed of light, and $d \Omega$ is the differential solid angle. Combining the above two equations and separating the scalar and vector quantities, the complete transition rate for the Raman scattered Stokes radiation is written as

$$
W=\frac{V \omega_{s}^{2} e^{4}}{4 \pi^{2} \hbar^{2} c^{3}}\left|E\left(\omega_{i}\right)\right|^{2}\left|E\left(\omega_{s}\right)\right|^{2}\left|\frac{\left\langle i, 1\left|\overrightarrow{\mathbf{r}} \cdot \hat{\boldsymbol{\varepsilon}}_{s}\right| m, 1\right\rangle\left\langle m, 1\left|H_{e v}\right| m, 0\right\rangle\left\langle m, 0\left|\overrightarrow{\mathbf{r}} \cdot \hat{\boldsymbol{\varepsilon}}_{i}\right| i, 0\right\rangle}{\left(E_{m}-E_{i}-\hbar \omega_{i}\right)\left(E_{m}-E_{i}-\hbar \omega_{s}\right)}\right|^{2} d \Omega
$$

where $\hat{\boldsymbol{\varepsilon}}_{i}$ and $\hat{\boldsymbol{\varepsilon}}_{s}$ are the polarizations of the incident and scattered photon.

The scattering cross section from a single molecule can then be obtained from the transition rate $W$. The photon flux through a given unit volume is

$$
\frac{V}{c}
$$


where $V$ is the unit volume and $c$ is the speed of light. For a single photon, the energy can be written in terms of the electric field and volume by

$$
|E(\omega)|^{2} V=\hbar \omega
$$

where $|E(\omega)|^{2}$ is the intensity of the incident photon.

Combining these two relationships with the transition rate equation, the differential scattering cross section for a Stokes shifted photon is written as

$$
\frac{d \sigma}{d \Omega}=\frac{\omega_{s}^{3} \omega_{i} e^{4}}{4 \pi^{2} c^{4}}\left|\frac{\left\langle i, 1\left|\overrightarrow{\mathbf{r}} \cdot \hat{\boldsymbol{\varepsilon}}_{s}\right| m, 1\right\rangle\left\langle m, 1\left|H_{e v}\right| m, 0\right\rangle\left\langle m, 0\left|\overrightarrow{\mathbf{r}} \cdot \hat{\varepsilon}_{i}\right| i, 0\right\rangle}{\left(E_{m}-E_{i}-\hbar \omega_{i}\right)\left(E_{m}-E_{i}-\hbar \omega_{s}\right)}\right|^{2}
$$

The scattering cross section can be shown from the above equation to be on the order of $10^{-28}$ to $10^{-32} \mathrm{~cm}^{2}$. The well known $\omega^{4}$ frequency dependence of normal Raman scattering is also evident in the above formula.

A schematic representation of surface enhanced Raman scattering from molecules adsorbed to nanometer sized metal particles is shown in Figure 7. Under certain conditions, the incident electric field of the 
photon can interact with the surface electrons of the metal and produce a surface plasmon resonance. Physically, the propagating plane wave of the photon has been localized to the surface of the metal particle. This electric field is highly localized near the surface of the metal extending approximately one half wavelength from the surface. [33]

The equation for the Raman transition rate illustrates the sensitivity of surface enhancement from plasmon resonances. Since the enhancement scales as the fourth power of the electric field, very large local field intensities are obtainable. In addition, if the physical system provides a favorable contribution to the electron-molecule Hamiltonian, further enhancements can be realized.

For smooth metal films, theoretical predictions of experimental measurements of surface enhancements are in good agreement. [31, 33] These values are in the range $10^{4}$ to $10^{6}$. Theoretical analyses for smooth interfaces are derived from the solutions of Maxwells equations at the interface of two dielectrics. [31, 33, 38] For small metal particles, Mie scattering is developed containing a system of multiple particles. Experiments performed utilizing both atomic force microscopy and SERS have shown that larger enhancements up to $10^{16}$ have been realized when multiple particles are aggregated in close proximity to each other. [26] Colloidal silver particles in solution represent and define an interface between two phases. The solid phase of the silver particles and 
liquid phase of their solute molecules experience a rearrangement of their respective surface charges when in contact. In fact, any phase, even a pure metal in vacuo, will produce a redistribution of charge at the surface. In the case of a metal, the free surface electrons will reorient themselves as to produce a negative outer charge layer. This negative charge layer protrudes slightly away from the bulk material, leaving an inner positive layer producing in effect a surface of dipoles.

Similarly, when the two phases of the colloidal solution come in contact, there is a tendency for the solution ions to orient themselves, in varying degrees, to the silver surface dipoles. As a result, an interior potential difference is created at the interior surfaces, and is known as the Galvani potential.

When a weak acid such as pMBA is dissolved in water, conjugate ionization does not go to completion. The degree to which the molecule is ionized is described by its acid dissociation constant, $K_{a .}[51]$. The weak acid equilibrium is described by

$$
\mathrm{HA} \stackrel{\mathbf{K}_{\boldsymbol{c}}}{\rightleftharpoons} \mathrm{H}^{+}+\mathrm{A}^{-} .
$$

The acid dissociation constant is described by the product of the concentrations of the ionized products relative to the neutral molecule and has the form 


$$
K_{a}=\frac{\left[H^{+}\right]\left[A^{-}\right]}{[H A]} .
$$

Figure 5 shows a schematic representation of the double layer potential. The effect of the double layer results in a shielding of the bulk solution ions from the reoriented surface charge. The measured $\mathrm{pKa}$ of the surface using SERS will not be the same as the pKa measurement of the bulk solution.

Using the well known theory of Guoy-Chapman for the diffuse double layer [22], the surface potential is related to the bulk potential by

$$
p H_{\text {Sufface }}=p H_{B u l k}+\left(\frac{e}{2.3 k T}\right) \Psi
$$

where $\Psi$ is the double layer potential difference. 


\section{Experimental Apparatus}

The experimental apparatus is shown in Figure 2. Excitation light at 514 nanometers $(\mathrm{nm})$ was produced by an argon ion laser (Coherent Innova 90) and sent through a $50 \mathrm{~nm}$ bandpass filter to remove unwanted fluorescence from the laser discharge tube. The beam was then focused with a $75 \mathrm{~mm}$ anti-reflection (AR) coated crown glass lens into the sample cuvette. The beam diameter at the lens was approximately $2 \mathrm{~mm}$. The laser was then terminated with a ThorLabs Black Hole beam dump.

Raman scattered light was collected by first placing an $\mathrm{f} / 1$ cylindrical lens to collimate the excitation volume. The collimated scattered light was sent through a holographic notch filter from Kaiser Optical having a $60 \mathrm{db}$ rejection ratio to block unwanted Rayleigh scattered laser light. The collimated scattered light was then focused onto the entrance slits of an Acton Research 300i spectrometer (f/\# 4) using a cylindrical lens of the same $f$-number $(f / \#)$ as the spectrometer. An Andor Model 465 EMCCD was used to collect the dispersed scattered light. The spectral acquisition from the $\mathrm{CCD}$ and the spectrometer control was performed using the AndorSpec software. Spectral analysis and graphing was performed using Kaleidagraph. 
The spectrometer was calibrated using reagent grade toluene. A sample calibration spectrum is shown in Figure 3. Toluene calibrations were performed each day before data acquisition to ensure wavelength accuracy and proper camera performance.

In order to collect as much scattered Raman light as possible and to avoid unwanted stray excitation light from entering the spectrometer, careful alignment of components are required. In order to change samples quickly and preserve alignment, a great deal of care was taken for initial optical alignment. Incorrect alignment can also cause unwanted scattered light to enter the spectrometer. This can cause diffuse scattered light to illuminate the CCD uniformly and simulate detector noise. Specular reflections inside the spectrometer of the Rayleigh light can also illuminate the CCD simulating spectral features A standard optical table, without air floating capability, was used for all measurements. Two apertures were placed 1 meter apart along a set of holes with the center of the apertures at six inches. This defined the alignment of the pump beam to be at the height of six inches and along a set of holes. Standard optical posts were placed directly into the table to ensure alignment along the holes. An alignment laser, helium neon, used two turning mirrors to direct and align the two apertures and provide the exact alignment of the pump beam path. Alignment of the pump beam in this was allowed different pump lasers to be aligned very 
quickly simply by aligning them through the two apertures. The HeNe laser was kept in place with the use of a flipper mirror throughout the experiments to periodically check the alignment.

Halfway between the two pinholes, two more apertures were placed orthogonal to the main pump beam line. This provided an exact ninety degree path to direct the scattered light into the monochromator. The apertures were placed again along a set of holes orthogonal to the main beam path at the height of six inches. A mirror was placed to direct the pump beam light through these apertures. Now an exact beam path was established to align the monochromator. The turning mirror was left in place in order to align the spectrometer.

Once the path of the spectrometer was determined and defined, the entrance slits and the center of the grating were used for the next stage of alignment. The spectrometer was adjusted so the center of the slits was at a height of six inches. The location of the slits was approximately $30 \mathrm{~cm}$ from the excitation beam path. The spectrometer alignment light was then centered on the entrance slits and the center of the grating. Since the laser beam is much smaller than the size of the grating, the slit size was reduced to provide a single slit diffraction pattern. This allowed the multiple orders to provide enough horizontal separation to center the beam on the grating very accurately. The spectrometer was then mounted firmly onto the optical table and secured. 
In order to record the complete Raman spectra on each acquisition period, the exit slits of the monochromator were removed and replaced with a CCD camera. Since the spectrometer images the entrance slits to a conjugate exit plane, proper positioning of the CCD pixel array is necessary to achieve optimum resolution and line shape.

The CCD was an Andor Model 465 having 13 micron pixel width arranged in an array of 512 horizontally and 512 vertically. If the array is not positioned at the image plane, and thus out of focus, the image of the slits will be larger than actual. This will reduce the spectral resolution of the instrument since the slit images will blur into each other.

In order to place the camera at the image plane, the entrance slits were reduced to minimum, which is approximately 10 microns, and then illuminated with an unfocussed helium neon laser. The output of the CCD was monitored in real time using the Andor acquisition software. Adjustments on the spectrometer allowed the camera to be positioned in the optical axis. The camera was adjusted to minimize the spectral width of the helium neon laser line. This now positions the camera correctly at the image plane.

The next adjustment involved the rotation of the camera so the vertical dimension of the pixel array is correctly aligned with the input slits. If the camera is not at the proper angle, the dispersed light will hit 
the pixels asymmetrically, and therefore display a distorted output image. The correct angle is obtained by rotating the camera so again the spectral width is again narrow AND symmetric. Generally, spectral symmetry involves camera rotation adjustment and spectral width involves a focal plane adjustment.

The sample holder was positioned at the intersection of the alignment axes of the spectrometer and the laser. The holder contained a spring assembly to secure the cuvette in place. In order to align the optics with spectrometer optical axis, a source of scattered light was needed. This was accomplished by first placing the cuvette in the sample holder. A white business card was then cut to the diagonal dimension on the cuvette and inserted in this fashion. The collection optics were then inserted and the scattered light centered on the entrance slits.

Silver colloid solution was prepared using the citrate reduction method of Lee and Meisel. All glassware was thoroughly cleaned in a $1 \%$ Micro detergent solution before use. Deionized water having a resistivity of 18 megohms was used for all processes. To prepare the silver colloid, $100 \mathrm{mg}$ of silver nitrate was added to $500 \mathrm{~mL}$ of deionized water and brought to a very gentle boil with constant stirring. At this point, the temperature was reduced to approximately $90 \mathrm{C}$ to avoid extensive evaporation of the solution. To this heated solution, $10 \mathrm{~mL}$ of a $1 \%$ sodium citrate was then added dropwise. Heating and stirring continued 
for approximately 30 minutes. At this point, a visual film of silver formed on the inside of the glass beaker. This solution was then filtered with .2 micron disc filters to provide the standard colloidal solution.

A saturated solution of pMBA was prepared in methanol. This preparation provided a high ratio of analyte to solvent with concentrations in the $\mathrm{mM}$ range, as verified by separate concentrations dissolved to completion. Methanol was chosen as the solvent over ethanol to minimize spectral interferences.

Ionic strength adjustment was performed with sodium chloride, potassium chloride, or sodium perchlorate. For low ionic strength solutions of a few millimolar or less, sodium chloride was initially used. Initial experiments using $\mathrm{NaCl}$ required an incubation time to provide a reasonable SERS signal. Extensive aggregation occurred when concentrations close to $10 \mathrm{mM}$ were used causing the silver colloid to fall out of solution almost immediately. These aggregation effects were not as sensitive with potassium chloride. Using $\mathrm{KCl}$, a $1 \mathrm{mM}$ solution could be prepared without immediate precipitation of the colloid. Samples prepared in this way could be used for at least one full day. Also, no incubation time was necessary. Samples were used immediately following preparation.

In order to prepare reproducible SERS samples, the following procedure was necessary. The stock colloidal solution was diluted with 4 
parts DI water to a volume of $1 \mathrm{ml}$ in a standard $10 \mathrm{~mm} \times 10 \mathrm{~mm}$ cuvette. $50 \mu \mathrm{L}$ of the pMBA saturated solution was then added and thoroughly mixed using a vortex shaker. At this point, the ionic strength was adjusted. $10 \mu \mathrm{l}$ of a $500 \mathrm{mM}$ solution of $\mathrm{KCl}$ was added and thoroughly mixed again using the vortex shaker. The orange color of the colloid/pMBA mixture turned to a lime green as viewed with transmitted light. This final solution was then mixed again with a vortex shaker for a few seconds and used immediately for analysis.

Spectra were collected using the Andor signal acquisition software. Typically 100 acquisitions of a one second each were summed to increase signal to noise. Since the signal to noise improvement scales as the square root of the number of acquisitions, a factor of 10 improvement was achieved in this manner. Data was exported as text to Kaliedagraph for analysis and plotting. 


\section{Results and Discussion}

The ordinary Raman spectrum (ORS) of pMBA powder is shown in Figure 8. The peak at $2564 \mathrm{~cm}^{-1}$ shows the S-H stretching vibration of the thiol group. The carboxylate group is identified by the $\mathrm{C}=\mathrm{O}$ stretching vibration at $1623 \mathrm{~cm}^{-1}$, and the $\mathrm{C}-\mathrm{COOH}$ stretching modes at 801 and $1183 \mathrm{~cm}^{-1}$. The aromatic ring shows strong stretching features at 1098 and $1595 \mathrm{~cm}^{-1}$. The symmetric $\mathrm{C}-\mathrm{H}$ stretch is identified at 3065 $\mathrm{cm}^{-1}$.

Since the solubility of pMBA in water is very low, the ORS at acidic $\mathrm{pH}$ could not be obtained. The ORS of $\mathrm{pMBA}$ in water was taken at $\mathrm{pH}$ 6.5 after adjustment with $\mathrm{NaOH}$. The $\mathrm{S}-\mathrm{H}$ vibration is still present near $2564 \mathrm{~cm}^{-1}$, but has split into two features at 2537 and $2582 \mathrm{~cm}^{-1}$. The bending mode at $846 \mathrm{~cm}^{-1}$ and the stretching mode at $1387 \mathrm{~cm}^{-1}$ identifies the presence of the carboxylate ion COO-.

The S-H bond splitting at 2537 and $2582 \mathrm{~cm}^{-1}$ shows a different behavior than that reported by Lee et al for ortho substituted MBA. [22] It has been reported that oMBA in the solid form or in solution forms intramolecular hydrogen bonds between the thiol group and oxygen of the carboxyl group. [52] The spectra of oMBA powder show a splitting of the S-H vibration at 2517 and $2529 \mathrm{~cm}^{-1}$. This splitting is not seen in 
the powder spectra of pMBA. This might be a result of the favorable positioning of the ortho substituted ring. [22] In addition, Colthup has reported [52] that most carboxylic acids exist as the dimer, identified by a weak Raman line due to the $\mathrm{C}=\mathrm{O}$ stretch in the $1625-1687 \mathrm{~cm}^{-1}$ region. In solution, oMBA does not show the splitting of the S-H stretch at neutral $\mathrm{pH}$. The spectrum of pMBA at $\mathrm{pH} 6.5$ taken in this study does show the splitting of the thiol feature.

The dissociation constants (pKa) corresponding to the thiol and carboxyl group in oMBA have been measured to be 9.5 and 5.4 respectively. $[22,48]$ One would expect similar values for the para substituted form. Near neutral $\mathrm{pH}, \mathrm{pMBA}$ would exist as $\mathrm{p}$ mercaptobenzoate. The presence of the COO- in the ORS of pMBA at $\mathrm{pH}$ 6.5 is consistent with this assumption. At basic condition, pMBA should exist as the p-sulphidobenzoate. In oMBA at $\mathrm{pH} 13$, no $\mathrm{S}-\mathrm{H}$ vibration is seen. For pMBA at $\mathrm{pH} 12.6$, the $\mathrm{S}-\mathrm{H}$ vibration is still present without splitting. Only the $2537 \mathrm{~cm}^{-1}$ feature remains. This would indicate the pKa value for the thiol group in pMBA is above 12.6 , a very surprising result based on the similarity of these two molecules. It is interesting to note that the splitting of the $\mathrm{S}-\mathrm{H}$ vibration is nearly removed at $\mathrm{pH}$ 8.1.

The SERS spectrum of pMBA in silver colloid is shown in Figure 10. Shown are the end point spectra for the titrations at low ionic strength. Similar titration end points were obtained for the high ionic 
strength titrations. The SERS spectrum is similar to the ORS with two notable differences. First, the S-H stretching vibration is absent from the SERS spectra at all $\mathrm{pH}$ values and ionic strengths. This indicates that pMBA is dissociatively adsorbed onto the metal surface through the thiol group. This is in agreement with the general view that the pKa values of thiols are lowered on metal surfaces [48], and in the case of pMBA, an understatement. In the ORS spectra of pMBA, the $\mathrm{S}-\mathrm{H}$ vibration is seen at $\mathrm{pH}$ near 13 , and in the SERS spectrum is never seen even at $\mathrm{pH}$ values of 2. The second difference in the ORS and SERS spectra is the shifting of the COO- stretching frequency. It has been suggested that this could be a result of non-bonded COO-groups having a more vertical orientation to the surface. [22]

Production of the carboxylate ion COO- is seen at 848 and 1434 $\mathrm{cm}^{-1}$. It seems reasonable then that observing the production of these bands during titration could monitor the production of the carboxylate ion. This is in fact the case; however, spectral analysis was difficult due to the shifting of the COO- band during titration. Figure 11 show the development of the SERS spectra during titration at low ionic strength. The spectra have been normalized to the aromatic ring vibration at 1077 $\mathrm{cm}-1$. The titration curves were analyzed by monitoring the peak of the spectral feature. Both the $\mathrm{COO}$ - and $\mathrm{C}-\mathrm{COO}$ - vibrations were used for 
analysis to identify any possible systematic error introduced by a possible competing spectral feature.

Figure 12 shows the results of the titrations at both low and high ionic strengths. At low ionic strength, the observed pKa for the carboxyl hydrogen is in reasonable agreement with the value of 5.4 reported for oMBA. This value is also consistent with the observation that the $\mathrm{pH}$ required for solvation of pMBA in water is 6.4 . Therefore a value near 6 is assumed for pMBA.

Assuming the value of 6 for the pKa of the carboxylate ion, the data show a small effect of the double layer potential. This is consistent with the double layer potential as calculated by $\mathrm{Yu}$ [27] for the titration of mercaptopyridine in a $100 \mathrm{mM}$ ionic strength solution. For those measurements, a potential corresponding to a $\mathrm{pH}$ value of 1.2 was calculated. Adding this to the assumed value of 6.0 gives a measured pKa of 7.2 for the carboxylate ion of pMBA.

These measurements were also performed using a confocal Raman microscope on single or few silver particles. In these experiments, similar solutions with $100 \mathrm{mM}$ ionic strength were prepared and a single particle was selected for measurements of the SERS spectra. Figure 13 shows the accumulated spectra as a function of $\mathrm{pH}$. The variation in some of the weaker features illustrates the advantages of single particle versus bulk measurements. These features are most likely due a slight 
reorientation of the molecule on the surface, and thus manifest additional vibrational modes through the reorientation of the molecule relative to the plane of the particle surface. Specific conformation geometries on individual particles are separated from the ensemble nature of the colloidal measurements. These conformational differences are not observed to significantly perturb the features used to analyze the functional ionization properties of the carboxyl group.

Figure 14 shows the resulting titration for the single particles. Surprisingly, the measured pKa is similar to the bulk measurements at lower ionic strength. These single particle measurements at high $\mathrm{pH}$ differ significantly from the measurements in the bulk colloid at the same high $\mathrm{pH}$.

This discrepancy poses a significant problem if one wished to use single functionalized particles as microscopic $\mathrm{pH}$ detectors. Clearly, further systematic studies are warranted. At the present time, current data does reveal insight into the nature of the colloidal effects.

It is well established that the ionic strength of a solution alters the bulk $\mathrm{pH}$ due to the activity coefficients of the participating ions. [51] Since the same assay was used for both the colloidal and single particle measurements, ie, silver colloid concentration, analyte concentration, and ionic strength, any effect due to a difference in assay composition 
can be neglected. The only difference in these two measurements is the size of the aggregates sampled.

In the bulk measurements, the interaction region of the laser with the colloidal sample was a cylinder of 100 microns in diameter and $5 \mathrm{~mm}$ in length. This large volume would contain a mixture of particles, aggregates, and fractals. Buffer solutions containing phosphate ions are known to significantly alter the activity coefficients when adsorbed on metal surfaces. [51, 52] Michota has also observed enhanced ionization activity of pMBA on silver aggregates. [48] Although a thorough understanding of the aggregate enhanced ionization of pMBA on silver colloid continues to elude us, the results are reproducible and further studies are necessary. 


\section{v. Conclusions}

Measurement of surface $\mathrm{pH}$ of pMBA molecules adsorbed to silver surfaces has been performed. The relationship between the $\mathrm{pKa}$ measured at the silver surface and the pKa of the bulk solution is used to determine the potential of the double layer. The potential of the double layer has found to be dependent on ionic strength.

Measurements in bulk silver colloid containing sub-micron aggregates show significantly different surface ionization activity than measurements performed on single particles using a confocal Raman microscope. It is suggested that aggregation effects of the silver colloid play a role in altering the activity coefficients of pMBA on silver surfaces. Understanding the physics of these effects is necessary for the development of single particle sensors of biological or chemical environments.

The non-invasive technique of monitoring the ionization of the pMBA self assembled monolayer as a function of ionic strength and $\mathrm{pH}$ also provided detailed information regarding the morphology and conformation of pMBA on silver surfaces in real time. The confocal microscope used for these measurements was equipped with a flow cell to monitor the molecular ionization in real time. This same arrangement could also be used to monitor the growth of self-assembled monolayers in 
real time providing a rapid throughput system capable of efficient development of high-quality self-assembled monolayers. By monitoring the SERS spectra of the adsorbed molecular layer, conformation and chemical specificity properties could be obtained in real time. This information could be used to optimize the morphology and functionality of SAMs.

The SERS technique also provides several orders of magnitude of dynamic range due to the sensitivity of the technique. Since this is an optical, noninvasive process, the real time monitoring of chemical or biological weapons should be possible. Response time is limited only by the acquisition time of an adequate signal to noise. This technique could provide a rapid, real time detection of chemical and biological substances of interest to national security. 


\section{Figures}

1. Graphical representation of Raman scattering.

2. Experimental apparatus of Raman spectrometer.

3. Raman spectrum of neat toluene.

4. Extinction spectra of neat colloids.

5. Schematic representation of double layer potential.

6. Molecular structure of PMBA.

7. Raman scattering near colloidal metal particles.

8. Ordinary Raman spectra of PMBA powder.

9. Ordinary Raman spectra of PMBA in water.

10. SERS spectra of PMBA.

11. Conformation behavior of carboxylate ion.

12. Ionic strength effect on titration.

13. Carboxylate behavior during titration.

14. Single (few) particle titration. 

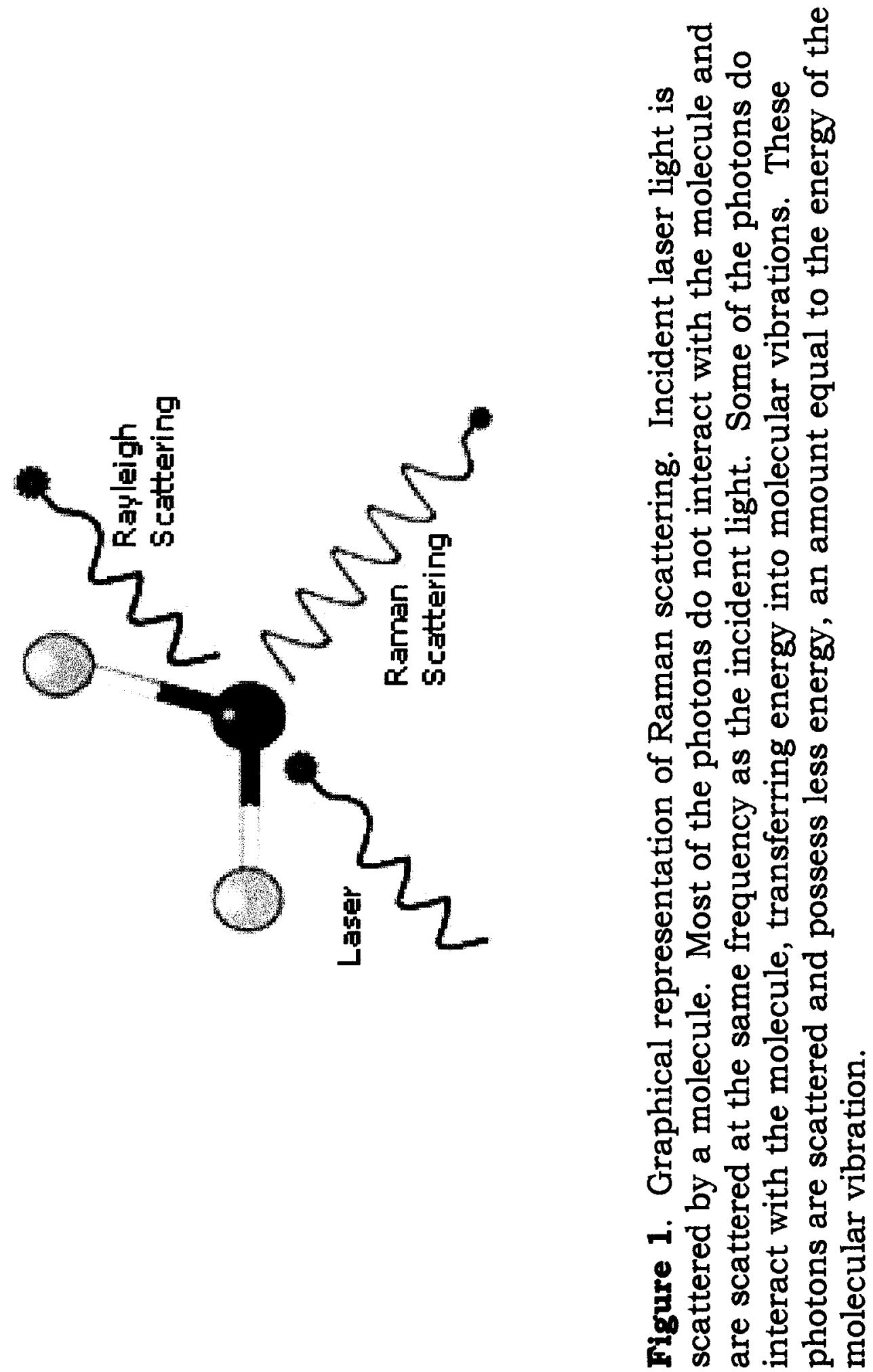


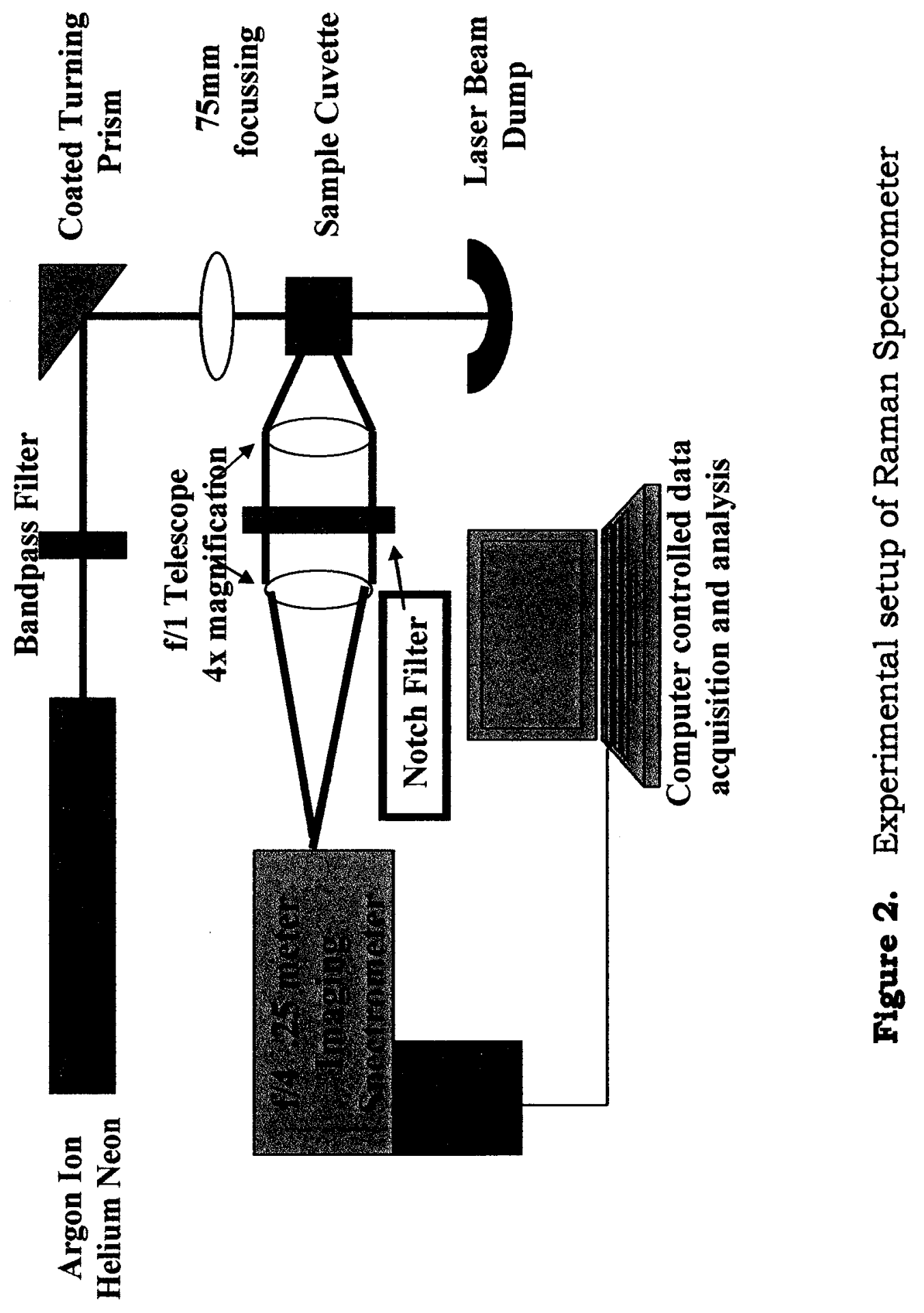




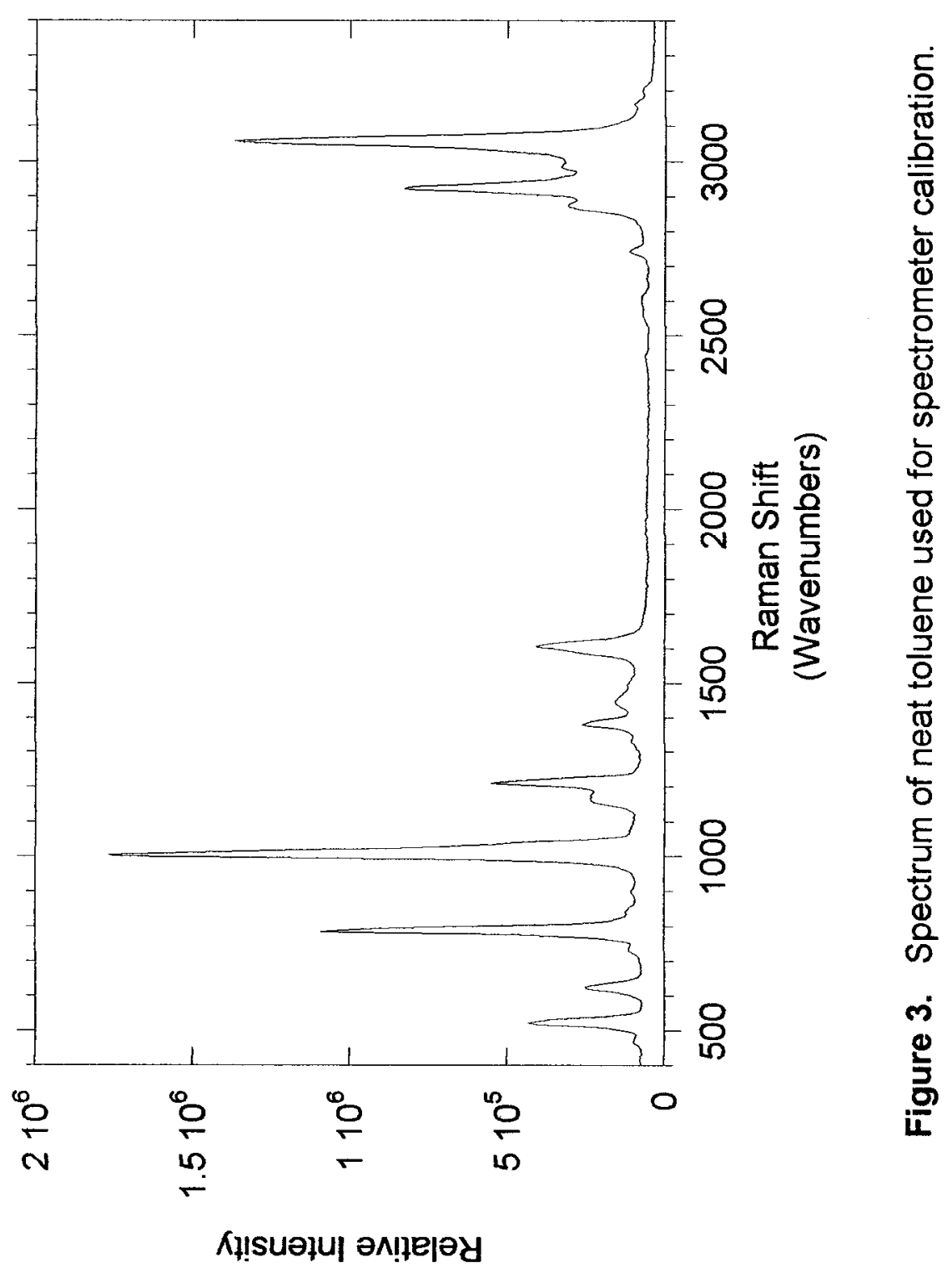




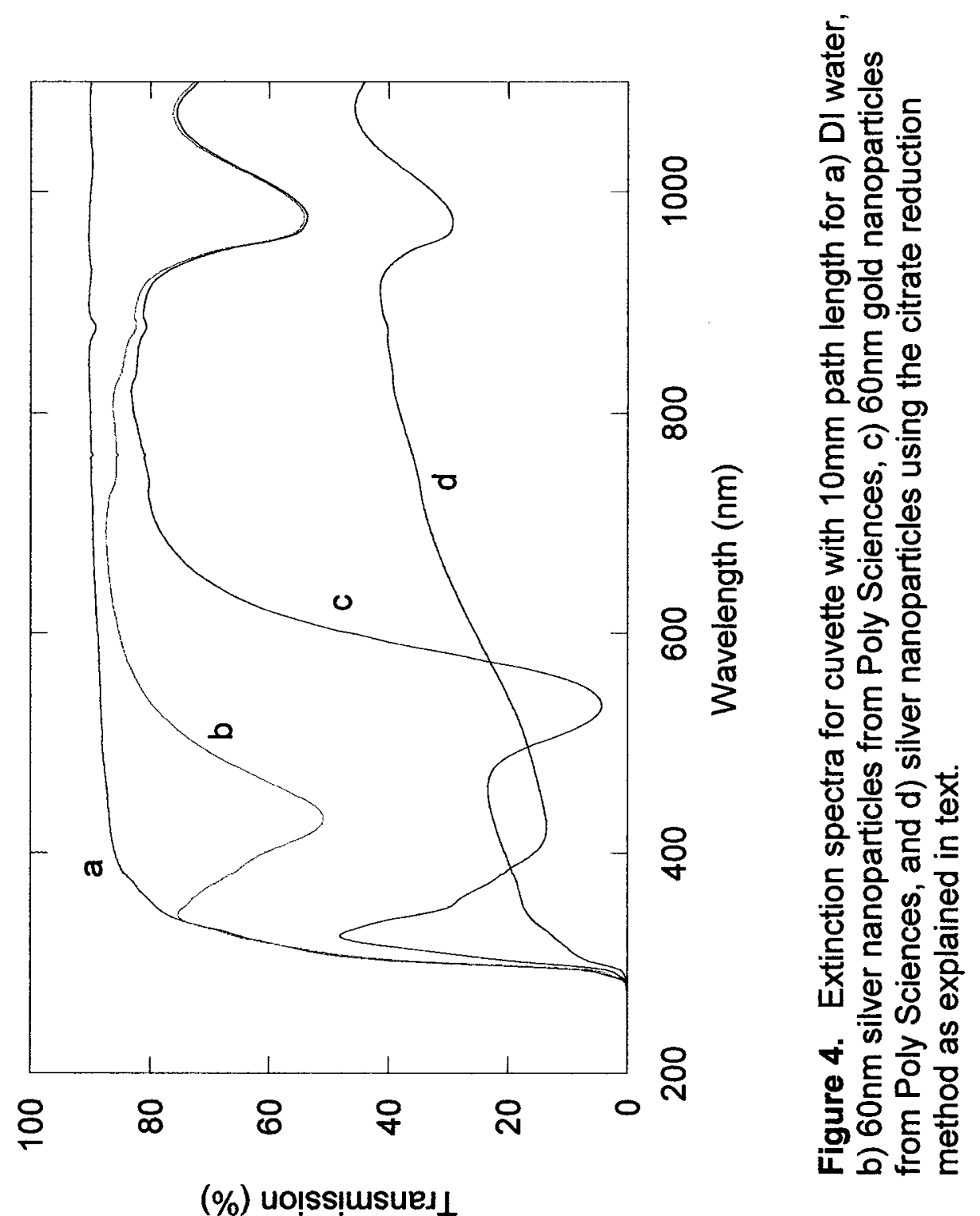




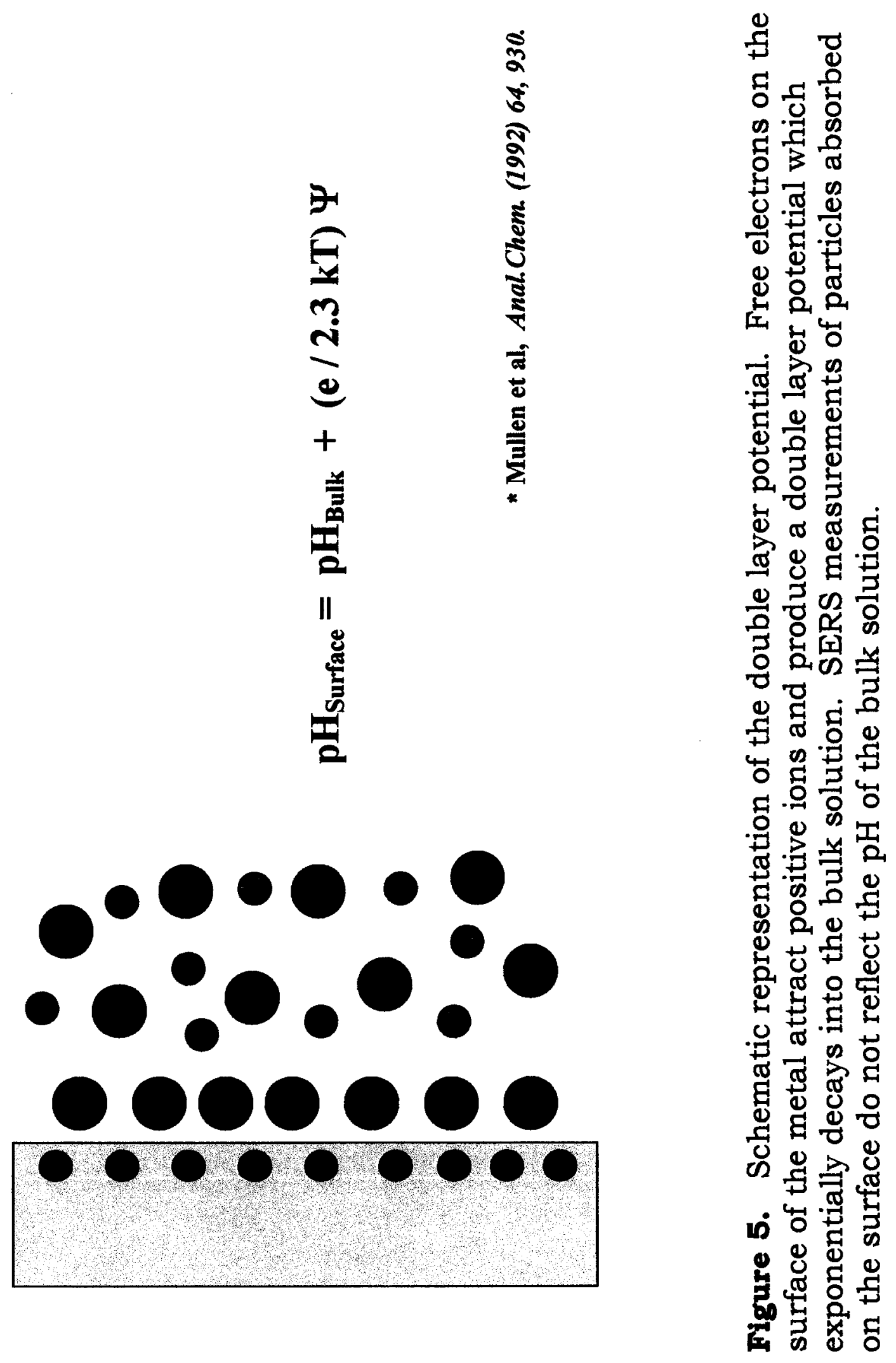



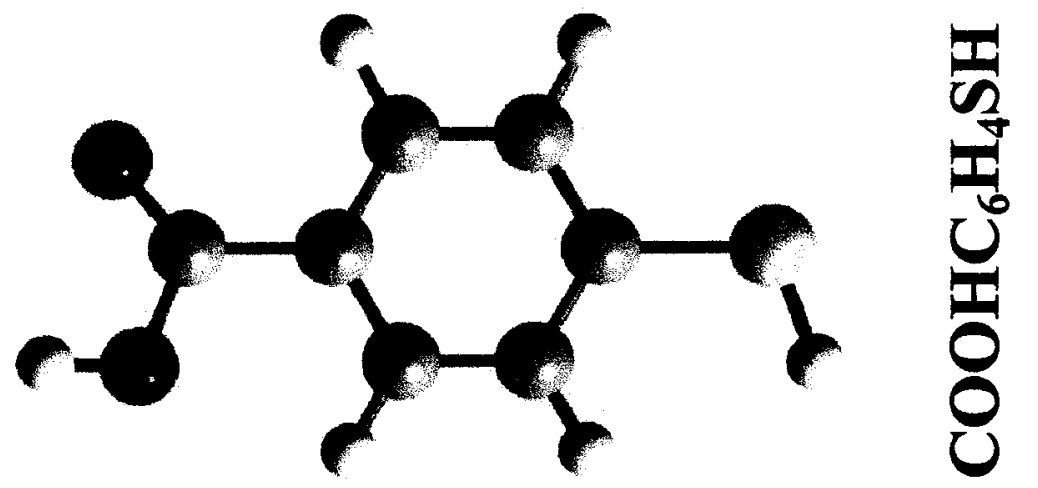



飐

욤

㖥
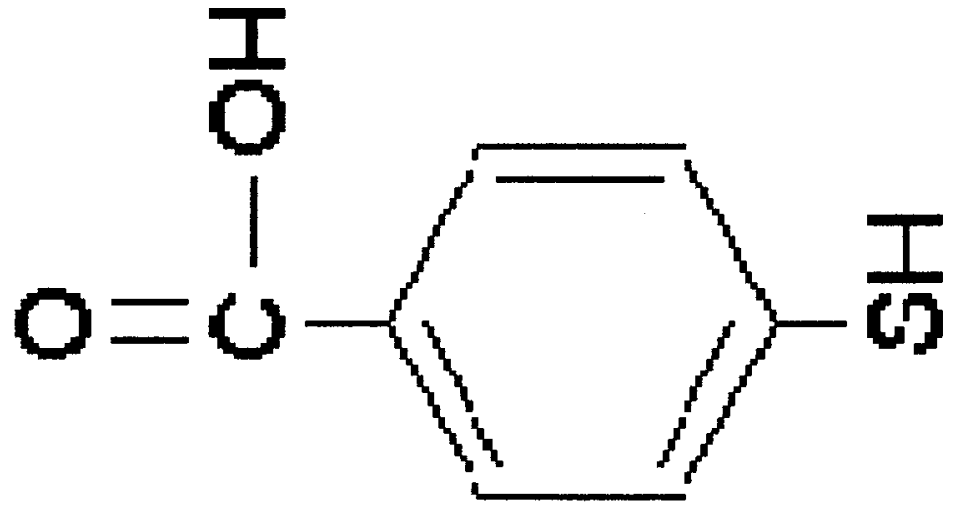

엄

呫

占

$\frac{0}{2}$

6

量 


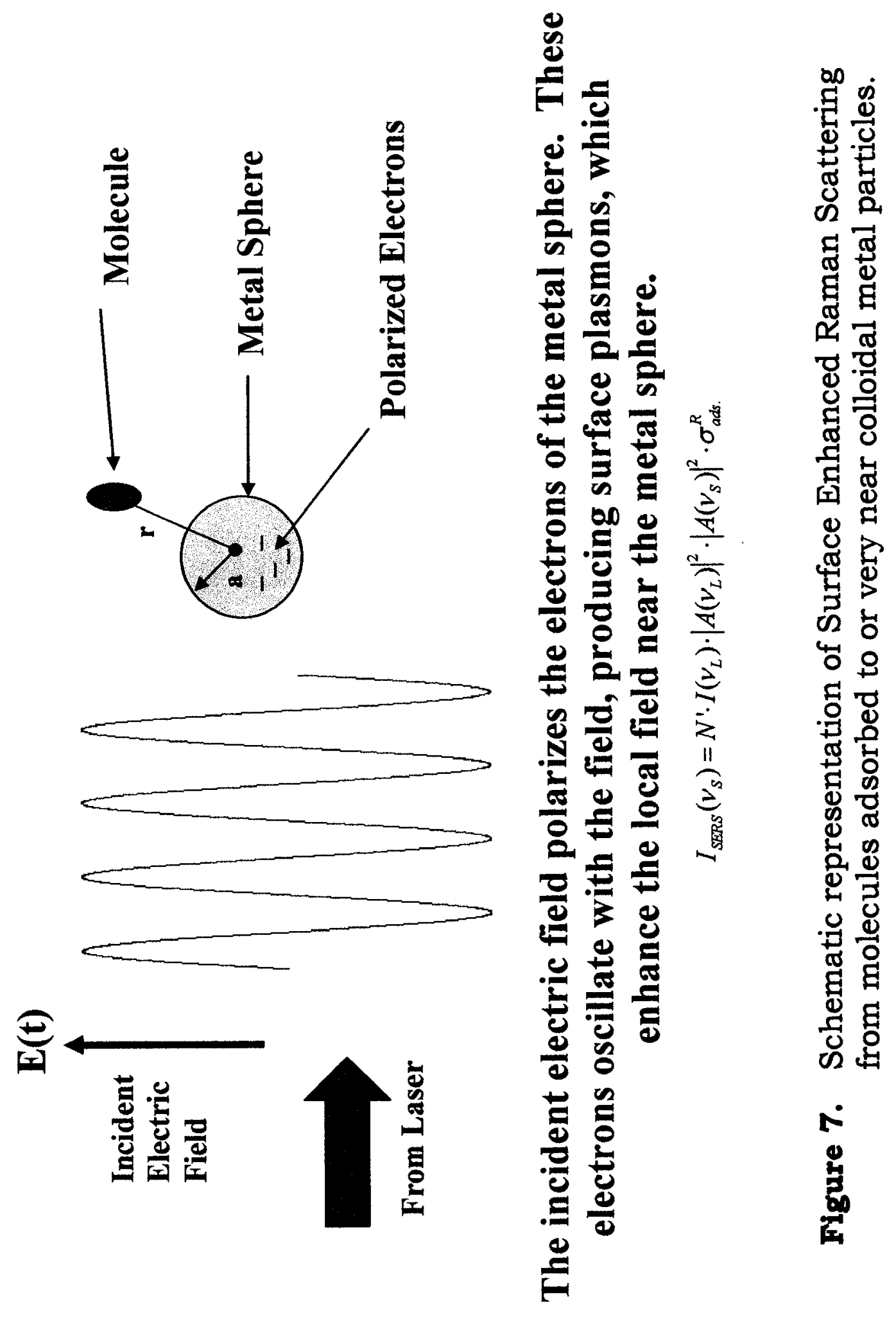




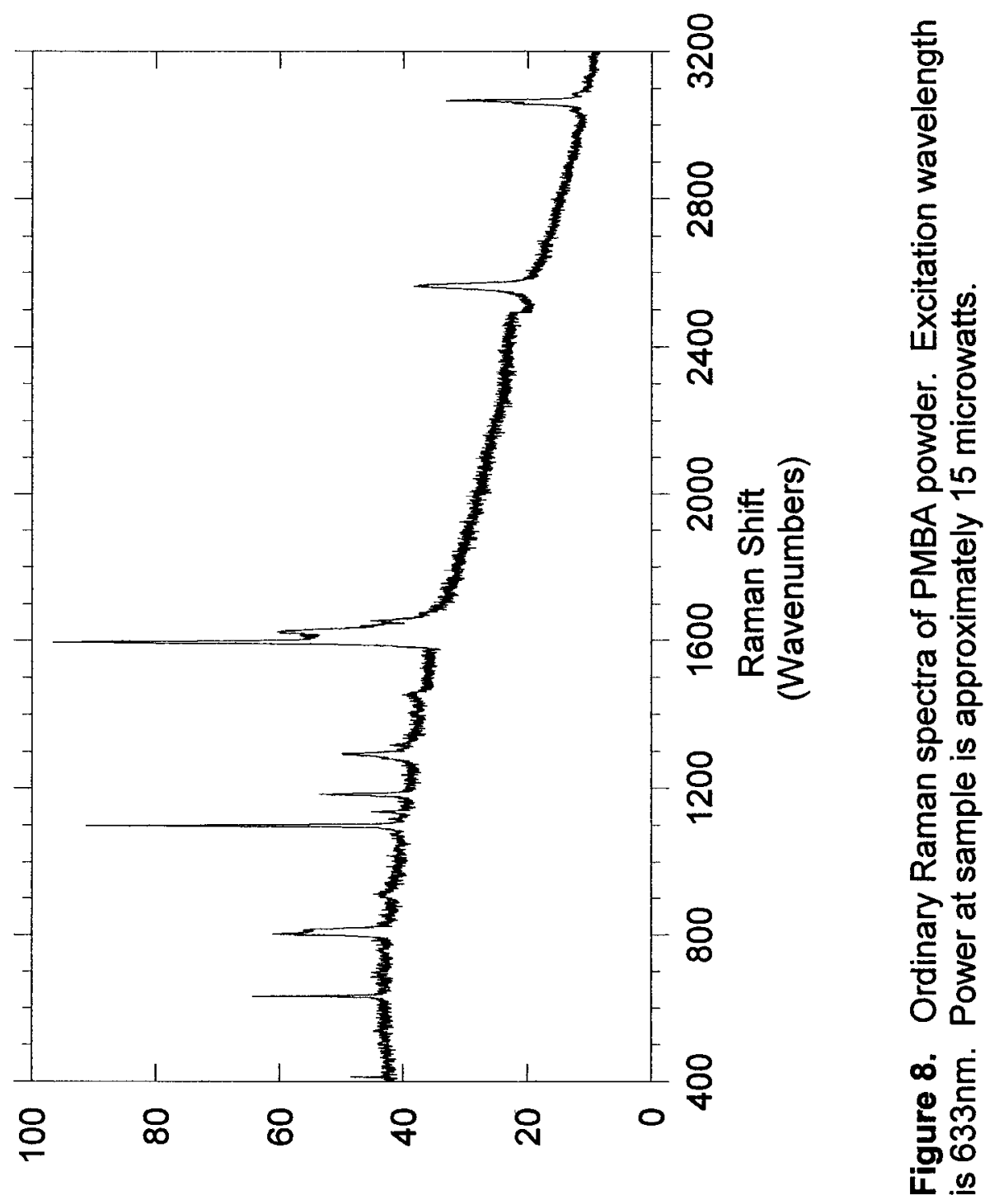

Кำsuәฺบ әм!ฺе|әу 


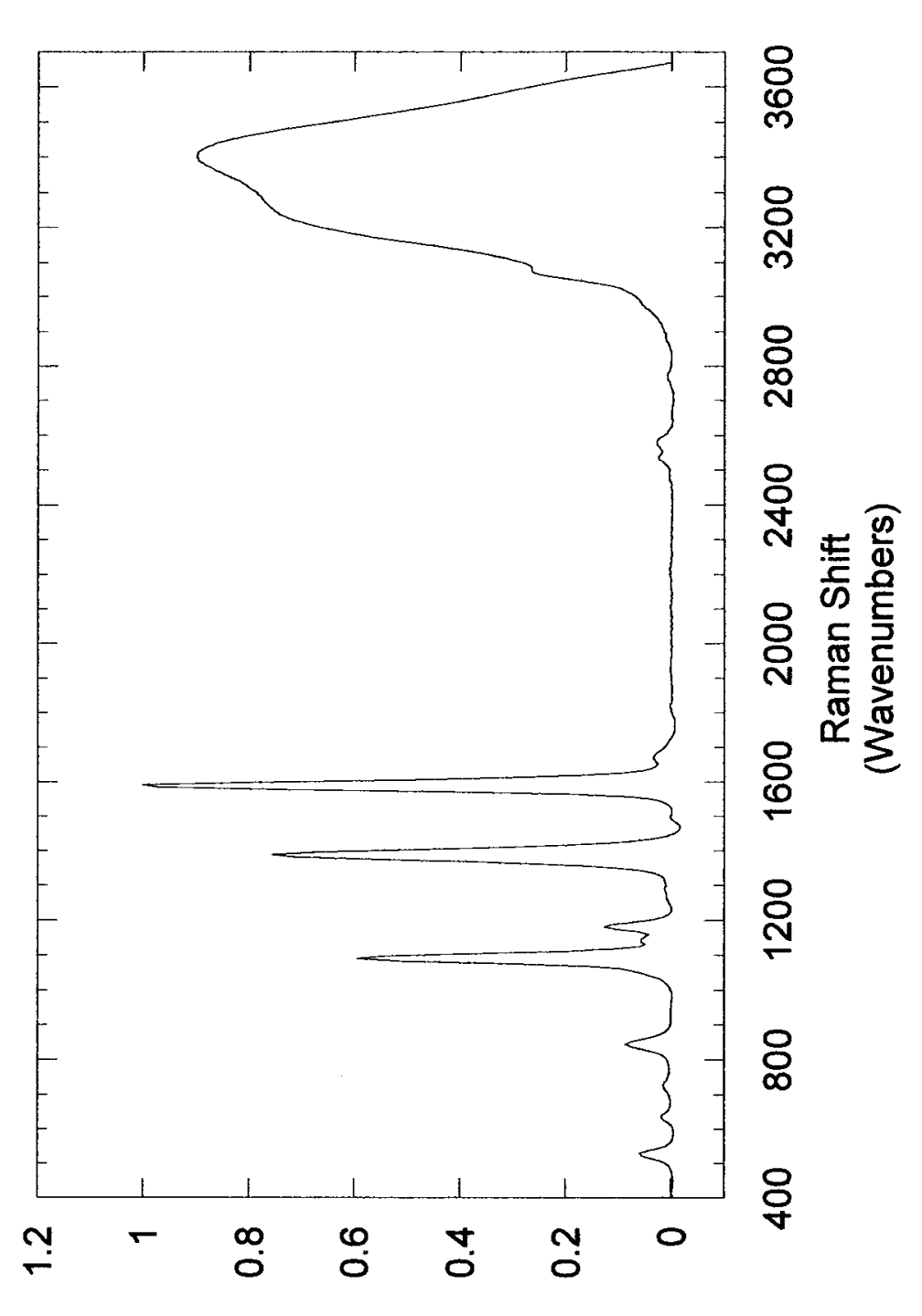

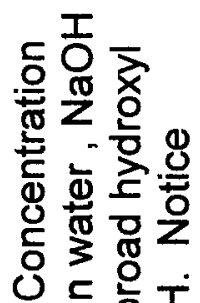

定要

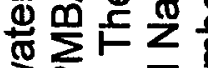

उ व

万茄要

.$\subseteq ?$

$<\overline{0}$

m글

$\sum_{0}$ 엉

政

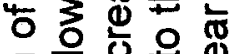

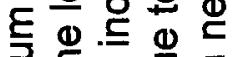

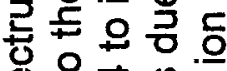

우요 응

00

도웡

त T元

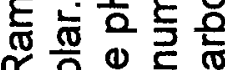
× 응 ᅯ $\geq \sum \pm 0$

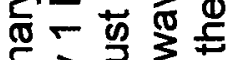

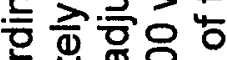
万苗

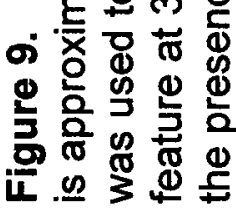
Kұ!suә̨u। pəz!!emoN 


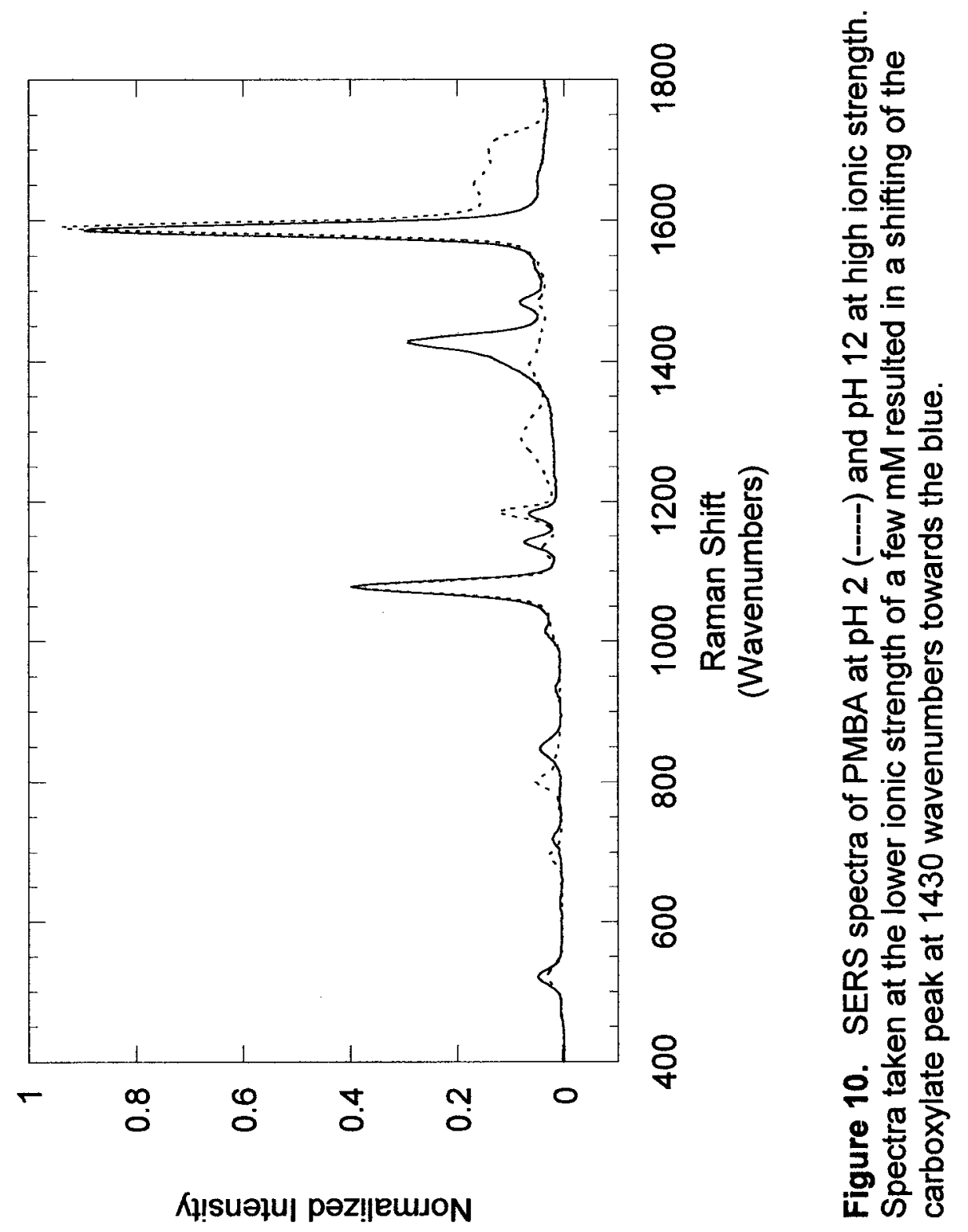



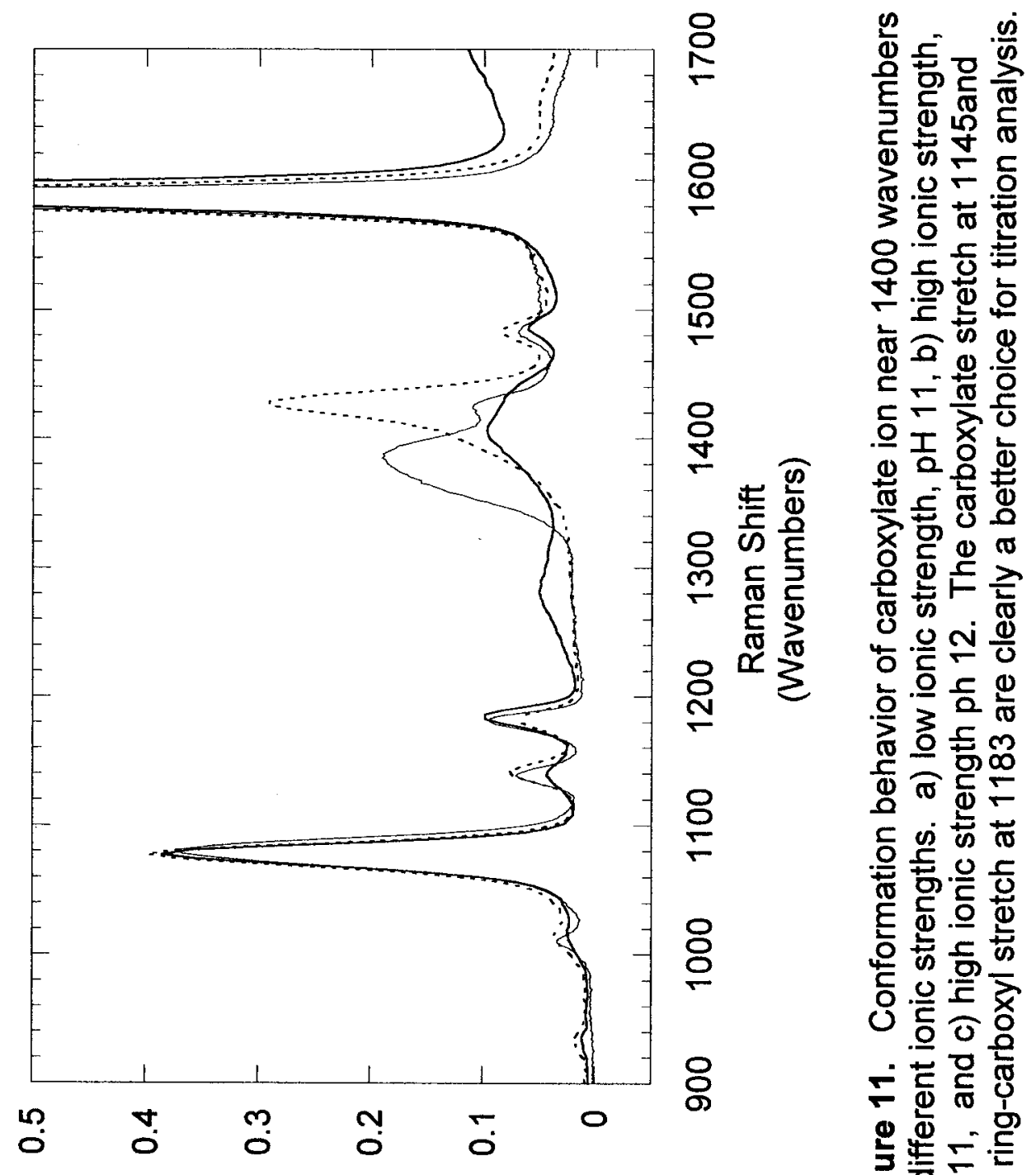

으읃언

늘 흔

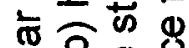

Ф2

ᄃ $=$ 훙요

드으 총ㄴำ

(4)

䒕 $=\frac{1}{0}$

爻苟

ㅇํㅇ 온

品紊什

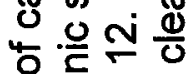

능 으 등 는

放兵

등

ర๐

등 复苗

은 苦.은

통 으 힌

은 严家

등 을 조

응ㅇㅇ

$\therefore$ 릉

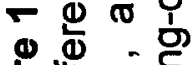

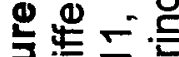

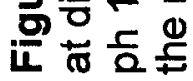

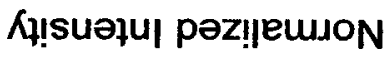




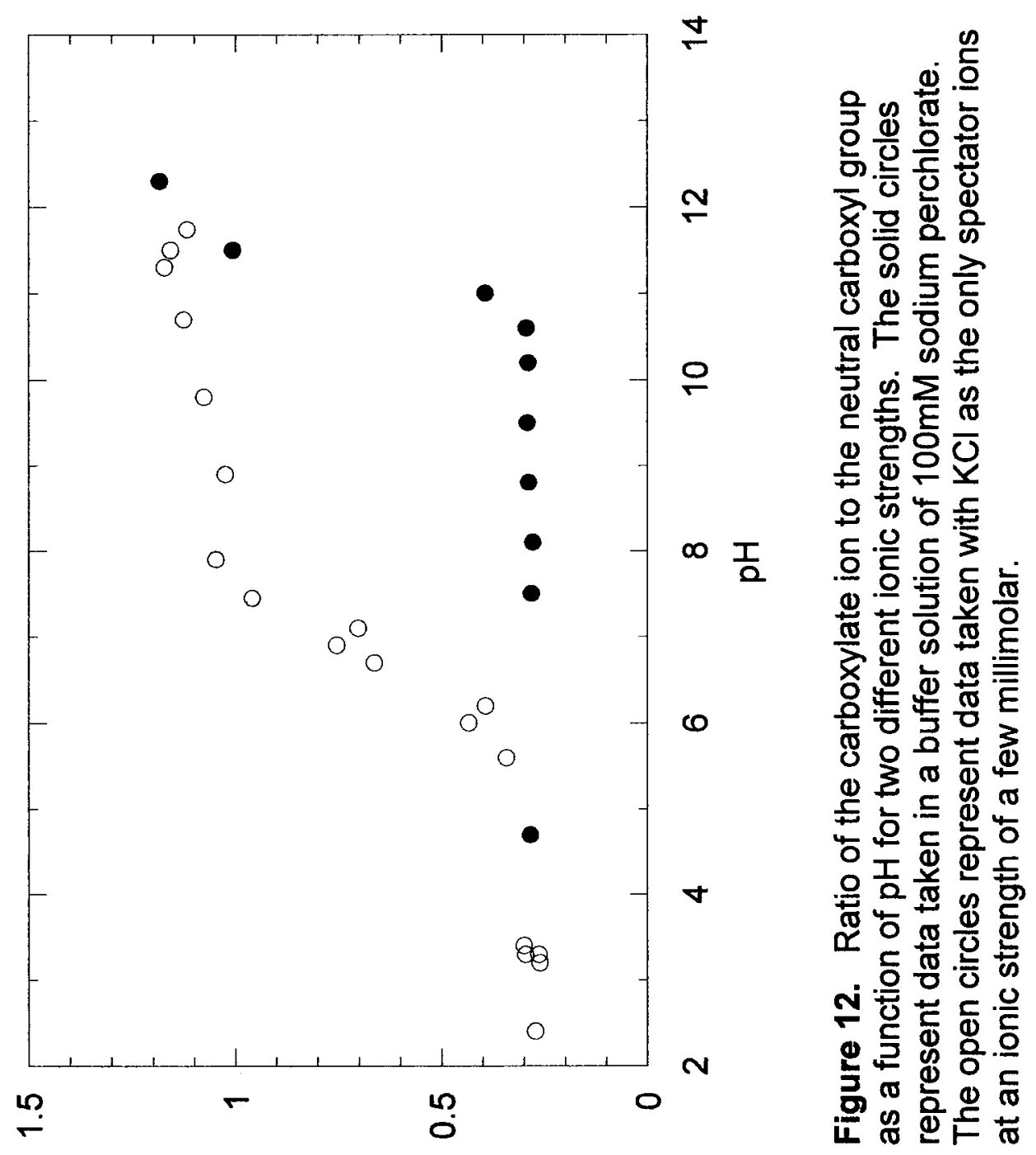

olfey ikxoques of əle|kxoques 


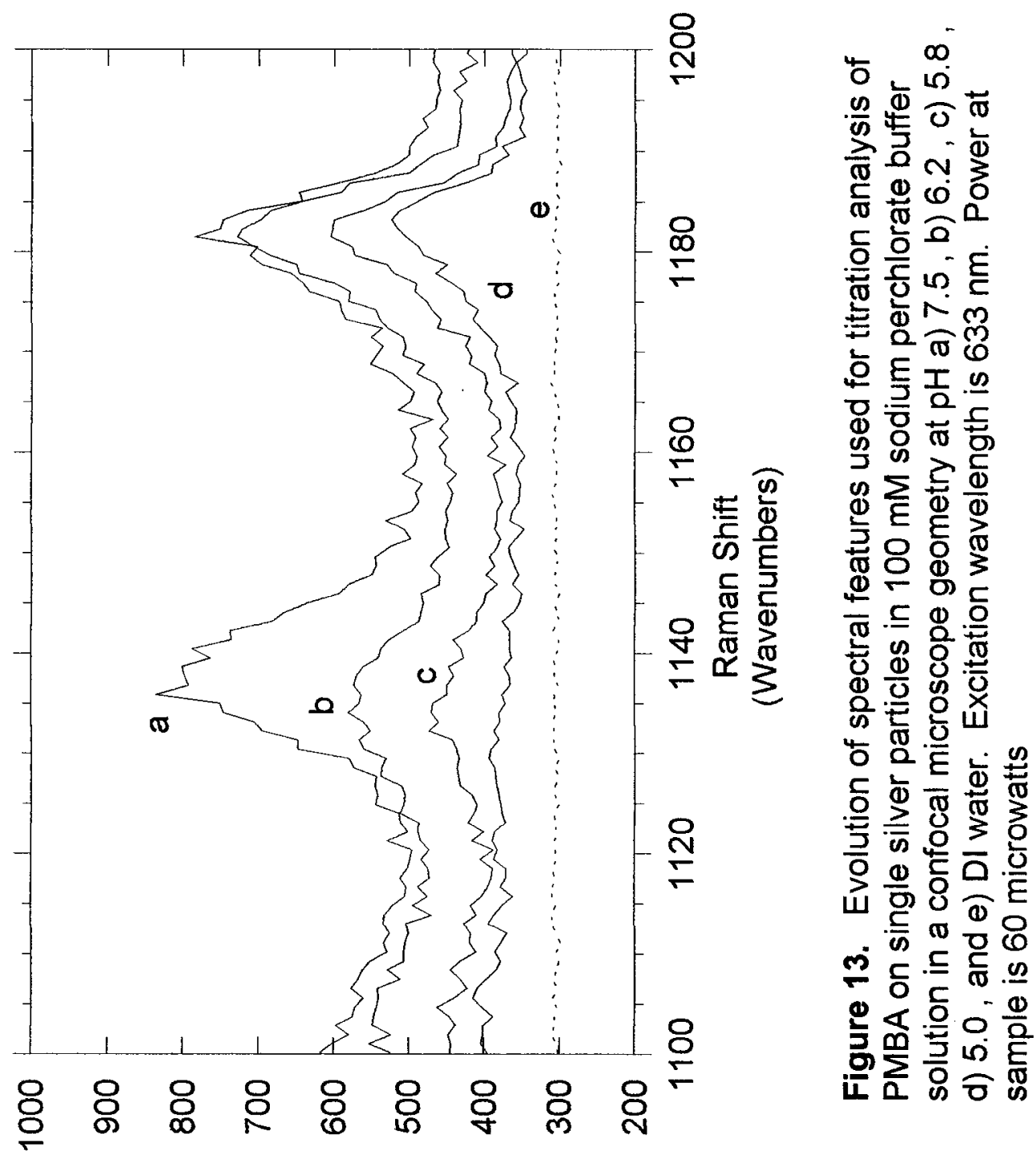

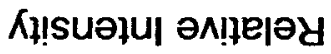




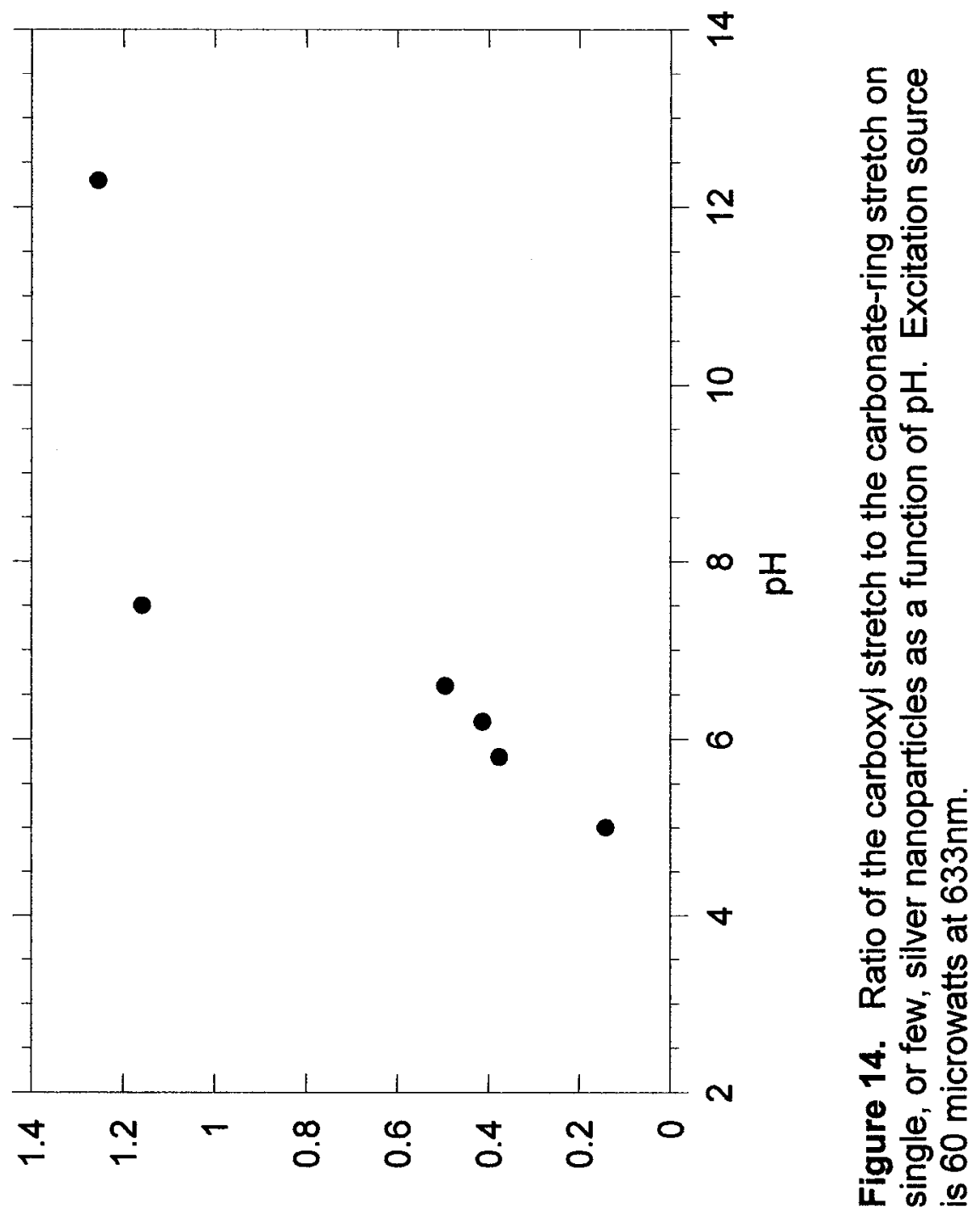

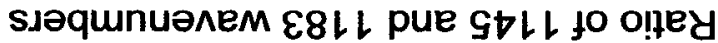




\section{References}

1. Bernath, P.F., Spectra of Atoms and Molecules. Topics in Physical Chemistry. 1995.

2. McCreery, R.L., Raman Spectroscopy for Chemical Analysis. 2000.

3. Raman, C.V. and K.S. Krishnan, A new type of secondary radiation. Nature, 1928. 121 : p. 501.

4. Ruf, T., Phonon Raman Scattering in Semiconductors, Quantum Wells, and Superlattices: Basic Results and Applications. Springer Tracts in Modern Physics. Vol. 142. 1998: Springer-Verlag.

5. Demtroder, W., Laser Spectroscopy. 2 ed. 1998.

6. Van Duyne, R.P., Chemical and Biochemical Applications of Lasers, ed. C.B. Moore. Vol. 4. 1979: Academic Press.

7. Campion, A. and P. Kambhampati, Surface enhanced Raman scattering. Chemical Society Reviews, 1998. 27: p. 241-250.

8. Kneipp, K., et al., Near infrared surface-enhanced resonance ranan scattering of trinitrotoluene on colloidal gold and silver. Spectrochimica Acta Part a-Molecular and Biomolecular Spectroscopy, 1995. 51: p. 2171.

9. Nie, S. and S.R. Emory, Probing single molecules and single nanoparticles by surface enhanced raman scattering. Science, 1997. 275(21 February 1997): p. 1102-1106.

10. Mathlouthi, M. and D.V. Luu, Laser raman spectra of d-fructose in aqueous solution. Carbohydrate Research, 1979. 78: p. 225-233.

11. Kacurakova, M. and M. Mathlouthi, FTIR and laser raman spectra of oligosaccharides in water: characterization of the glycosidic bond. Carbohydrate Research, 1996. 284: p. 145-157. 
12. Kneipp, K. and H. Kneipp, Detection and identification of a single DNA base molecule using surface-enhanced Raman scattering (SERS). Physical Review E, 1998. 57(6): p. 57.

13. Kneipp, K., et al., Ultrasensitive Chemical Analysis by Raman Spectroscopy. Chem. Rev., 1999. 99: p. 2957-2975.

14. Kneipp, K., et al., Surface-enhanced Raman scattering: A new tool for biomedical spectroscopy. Current Science, 1999. 77(7): p. 915.

15. Kanayama, N. and H. Kitano, Interfacial recognition of sugars by boronic acid carrying self assembled monolayer. Langmuir, 2000. 2000(16): p. 577-583.

16. McNichols, R.J. and G.L. Cote, Optical glucose sensing in biological fluids: an overview. Journal of Biomedical Optics, 2000. 5(1): p. 516.

17. Pappas, D., B.W. Smith, and J.D. Winefordner, Raman spectroscopy in bioanalysis. Talanta, 2000. 51(2000): p. 131-144.

18. Graham, D., et al., Surface enhanced resonance raman scattering (SERRS): A first example of its use in multiplex genotyping. Chemphyschen, 2001. 2001(12): p. 746-748.

19. Hildebrandt, P. and M. Stockburger, Surface enhanced resonance raman spectroscopy of Rhodamine $6 G$ adsorbed on colloidal silver. J. Chem. Phys, 1984. 1984(88): p. 5935-5944.

20. Fleischmann, P.J. and A.J. Hendra, Raman spectra of pyridine adsorbed at a silver electrode. Chemical Physics Letters, 1974.

21. Campion, A., Vibrational Spectroscopy of Molecules on Surfaces, ed. Y.a. Madey. 1987, New York, NY: Plenum.

22. Lee, S.B., K. Kim, and M.S.K. Kim, Surface Enhanced Raman Scattering of 2-Mercaptobenzoic Acid in Silver Sol. Journal of Raman Spectroscopy, 1991. 22: p. 811-817. 
23. Lawless, M.K. and R.A. Mathies, Excited state structure and electronic dephasing time of Nile Blue from absolute resonance raman intensities. J. Chem. Phys, 1992. 96(11): p. 8037-8045.

24. Kneipp, K., et al., Approach to single molecule detection using surface enhanced resonance raman scattering (SERRS): A study using rhodamine 6G on colloidal silver. Applied Spectroscopy, 1995. 49(6): p. $780-784$.

25. Emory, S.R. and S. Nie, Near Field Surface Enhanced Raman Spectroscopy on Single Silver Nanoparticles. Analytical Chemistry, 1997. 69: p. 2631.

26. Kneipp, K., et al., Extremely large enhancement factors in surface enhanced raman scattering for molecules on colloidal gold clusters. Applied Spectroscopy, 1998. 52(12): p. 1493-1497.

27. Yu, H.-Z., N. Xia, and Z.-F. Liu, SERS Titration of 4Mercaptophyridine Self-Assembled Monolayers at Aqueous Buffer/Gold Interfaces. Analytical Chemistry, 1999. 71: p. 13541358.

28. Kottmann, J. and O. Martin, Plasmon resonant coupling in metallic nanowires. Optics Express, 2001. 8(12): p. 655.

29. Kottmann, J. and O. Martin, Plasmon resonances of silver nanowires with a nonregular cross section. Physical Review B, 2001. 64: p. 235402.

30. Lee, P.C. and D. Meisel, Adsorption and surface enhanced raman of dyes of silver and gold sols. J. Phys. Chem., 1982. 1982(86): p. 3391-3395.

31. Arya, K. and R. Zeyher, Theory of surface-enhanced Raman scattering from molecules adsorbed at metal surfaces. Physical Review B, 1981. 24(4): p. 1852.

32. Arya, K., Electric field enhancement near a randomly rough metal surface: The effect of a dielectric overlayer. Physical Review B, 1984. 30(12): p. 7242 . 
33. Moskovits, M., Surface Enhanced Spectroscopy. Review of Modern Physics, 1985. 57: p. 783.

34. Arya, K., Anderson Localization of Electromagnetic Waves in a Dielectric Medium of Randomly Distributed Metal Particles. Physical Review Letters, 1986. 57(21): p. 2725.

35. Otto, A., Surface enhanced Raman Scattering. Journal of Physics: Condensed Matter, 1992. 4: p. 1213.

36. Kreibig, U. and M. Vollmer, Optical Properties of Metal Clusters. 1995, New York: Springer.

37. Garcia-Vidal, F.J. and J.B. Pendry, Collective theory for surface enhanced raman scattering. Physical Review Letters, 1996. 77(6): p. $1163-1166$.

38. Jackson, J.D., Classical Electrodynamics. 3rd ed. 1998, New York: Wiley.

39. Corni, S. and J. Tomasi, Surface enhanced Raman scattering from a single molecule adsorbed on a metal particle aggregate: A theoretical study. Journal of Chemical Physics, 2002. 116(3): p. 1156.

40. Otto, A., Journal of Raman Spectroscopy, 1991. 22: p. 743.

41. Michaels, A.M., M. Nirmal, and L.E. Brus, Surface enhanced raman spectroscopy of individual rhodamine $6 \mathrm{G}$ molecules on large $\mathrm{Ag}$ nanocrystals. J. Am. Chem. Soc., 1999. 1999(121): p. 9932-9939.

42. Kneipp, K., et al., Approach to Single Molecule Detection Using Surface-Enhanced Resonance Raman Scattering (SERRS): A Study Using Rhodamine $6 G$ on Colloidal Silver. Applied Spectroscopy, 1995. 49(6): p. 780.

43. Ha, T., Single Molecule Fluorescence Resonance Energy Transfer. Methods, 2001. 25: p. 78.

44. Dos Remedios, C. and P. Moens, Fluorescence Resonance Energy Transfer Spectroscopy is a reliable ruler for measuring structural 
changes in proteins. Journal of Structural Biolog, 1995. 115: p. 175.

45. Wei, A., et al., Tunable surface enhanced raman scattering from large gold nanoparticle arrays. Chemphyschen, 2001. 2001(12): p. 743-745.

46. Jin, R., et al., Photoinduced conversion of silver nanoshperes to nanoprisms. Science, 2001. 294: p. 1901-1903.

47. Nickel, U., et al., A silver colloid produced by reduction with hydrazine as support for highly sensitive surface enhanced raman spectroscopy. Langmuir, 2000. 2000(16): p. 9087-9091.

48. Michota, A. and J. Bukowska, Surface enhanced Raman scattering of 4-Mercaptobenzoic acid on silver and gold substrates. Journal of Raman Spectroscopy, 2003. 34: p. 21-25.

49. Qu, J.Y. and L. Shao, Near infrared raman instrument for rapid and quantitative measurements of clinically important analytes. Review of Scientific Instruments, 2001. 72(6): p. 2717-2723.

50. Kneipp, K., A.S. Haka, and H. Kneipp, Surface-enhanced Raman Spectroscopy in single living cells using gold nanoparticles. Applied Spectroscopy, 2002. 56: p. 150.

51. Harris, D.C., Quantitative Chemical Analysis. 5th ed. 2000: W. H. Freeman.

52. Lin-Vien, D., et al., The Handbook of Infrared and Raman Frequencies of Organic Molecules. 1991: Academic Press. 